\title{
IL PORFIDO E LE SITUAZIONI DEL POTERE NORMANNO DI FRONTE ALL'EVENTO MORTE. SOCIOLOGIA DI UN SEGNO DEL RANGO NEL MEDIOEVO
}

\author{
Antonio Pio Di Cosmo \\ Universidad de Córdoba
}

\begin{tabular}{ll}
\hline Recibido: 09/10/2017 & Aceptado: 06/06/2018 \\
Revisado: 01/06/2018 & Publicado: 29/06/2018
\end{tabular}

RIASSUNTO

La descrizione della fenomenologia del potere rimanda ad un'attività semiologica volta all'affermazione ed alla distinzione del rappresentante dello Stato. Un'attività che è prima di tutto culturale, diretta a comunicare i valori dell'istituzione, della forza militare e magica del suo rappresentante, che anche nella morte mostra dei segni che suscitano reverenza. Proprio la rappresentazione di questi segni del sovrano rinvia ad un circuito simbolico, la cui efficacia ideologica è dimostrata dalla forza persuasiva che questi segni dimostrano. Altrettanto convincenti devono apparire le spazialità in cui il corpo di chi detiene il potere va conservato.

Il contributo analizza il ruolo giocato dal porfido nel funerale regio siciliano. Questa ricerca applica le conoscenze in materia archeologica, antropologica e storica, per raccontare l'azione della corte siciliana, che risolve le questioni circa i problemi di rappresentazione del sovrano defunto rispetto all'istituzione che rappresenta. In questo modo si vagliano le strategie di comunicazione orientate al timor reverentiae che dirigono le scelte dei sovrani normanni. S'apre così ad un nuovo orizzonte di ricerca per la fenomenologia del dolore partendo dal caso specifico del costume funerario dei re siciliani.

Parole chiave

Re di Sicilia; Funerale regio; Status symbol; Sepolcro; Porfido.
RESUMEN

La descripción de la fenomenología del poder se refiere a una actividad semiológica dirigida a la afirmación y distinción del representante del Estado. Una actividad principalmente cultural, destinada a comunicar los valores de la institución, la fuerza militar y mágica de su representante, tampoco el rey muerto tene que tener signos que estimulan reverencia. La misma representación de estos signos del soberano se refiere a un circuito simbólico, cuya efectividad ideológica tene que ser demostrada por la fuerza persuasiva que estos signos muestran. Igualmente convincente debe aparecer la espacialidad en la que se debe preservar el cuerpo de aquellos que tienen el poder. Este trabajo plantea el papel de el pórfido en el funeral de los reyes sicilianos. La investigación utiliza el conocimiento de la arqueología, de la antropología y de la historia, que se pueden aplicar a la obra de la corte real siciliana, esto concluye los problemas de representación de los reyes sicilianos falecidos, que están en fruente a la institución que los mismos representan. De esta manera se profundiza las estrategias de comunicación del timor reverentiae, que orientan las opciones de los soberanos normandos. Con todo ello se da forma a un nuevo método de aproximación a la fenomenología de el dolor a patir de el caso específico a cerca de el constumbre funerarios de los reyes sicilianos.

Palabras clave

Rey de Sicilia; Funeral real; Status symbol; Sepulcro; Pórfido.

apiocosmo@outlook.it 
«... e dentro l'urne confortate di pianto è forse il sonno della morte men duro? (...) e quando vaghe di lusinghe innanzi a me non danzeran l'ore future». (Ugo Foscolo, I sepolcri, 1-2;4-5.)

Il presente contributo tenta di far chiarezza su una funzione mai sufficientemente indagata del porfido: il valore di status symbol. Questa pietra viene adoperata anche dai sovrani normanno-svevi a coronamento dei "riti di espulsione" del re dalla società. Il prezioso marmo diventa così «riserva simbolica» e prerogativa "giuridica» e "tecnica» della monarchia locale (Gandolfo 2003). Uno degli essentialia del "Teatro del potere" insomma. Il marmo assurge dunque a segno del rango, specie in un periodo in cui costituisce un vero e proprio lusso. Anna Comnena lo sa bene, perché questo nel XI sec. diventa una velleità che nemmeno i basileis possono permettersi. I papi al contrario ne hanno gran disposizione nelle emergenze romane, che riutilizzano per le proprie sepolture, come dono diplomatico o ne fanno uno dei commerci più redditizi dell'Età Media. Pertanto i normanni, in un periodo di floridezza del suo commercio, riescono ad appropriarsi di quantità discrete di questa pietra fino a renderla una prerogativa del corredo simbolico della propria esperienza monarchica. Una possibilità d'approvvigionamento che però non durerà a lungo.

1. Il PORFIDO: UN LEMMA INDISPENSABILE PER “LEGGERE” IL “TEATRO DEL POTERE”

L'indagine procede dall'alta considerazione di questo marmo, quale strumento gnoseologico per comprendere la réclame normanna. La sua scelta risponde ad un' «attività semiologica» e "culturale», che illumina i passaggi con cui si struttura il volto pubblico della monarchia siciliana. Una riflessione che, nonostante l'abbondante letteratura sul tema, apre ad una rivisitazione delle strategie di rappresentazione della regalità locale e risale alle strutture dell'universo mentale dell'uomo medievale. Il porfido allora può essere considerato un lemma di un discorso complesso, che si esprime attraverso i simboli, quali protagonisti dei "sistemi rappresentativi» della realtà (De Champeux, 1992, 55). Assurge a segno che esterna il rango, ne fonda l'autorevolezza e col suo sentire va ben oltre le prerogative giuridiche proprie di un re. Tale percezione aumenta esponenzialmente per l'uomo medio del Medioevo, al- lorquando i formalismi hanno un valore sostanziale ed i fruitori sono molto attenti ai segni esteriori.

Il porfido, quale segno d'eccellenza in se e per sé, crea "gerarchie di supremazia", induce alla deferenza e rende impensabile ogni tentativo di sovversione dell'ordine costituito, conferendo alla formula di governo l'autorevolezza dell'investitura divina (Carile, 2002, 53). Un sentire, che se pur appartiene all'alea della maestà imperiale, può essere applicato non senza contraddizione alla ben più ristretta autorità regia. Un significato, quello attribuito dal medioevo al marmo, che non va cercato solo nei lapidari o nella letteratura di genere come il " $D e$ gemmis" di Epifanio di Salamina, le "Etymologiae" di Isidoro di Siviglia o il "De mineralibus" di Alberto Magno, dove non trova il giusto spazio; a tal fine non è sufficiente nemmeno la retorica uffciale (Tronzo, 2011, 8).

Il segno di status va letto nel contesto sociologicamente e culturalmente determinato del "Teatro del potere”. Innanzi alla cesura causata dall'evento morte si consuma poi l'assolutizzazione dei segni del rango, che potenzia l'autorevolezza della monarchia. La scelta della nuance e del dettaglio prezioso non è altro che il tentativo d'ottimizzare gli effetti della falsificazione di un codice. Una simile operazione presuppone il perdurare di una coscienza simbolica locale, che giustifica le condotte e chi le pone in essere. La connessione tra marmo e sovrano diviene intenzionale, perché suscita la visualizzazione dell'idea ed ha nell'immanente finalità "protettive" (Tronzo, 2011, 20-21). La funzione meramente visuale si realizza nell'identificazione fra contenitore e contenuto, tanto da costituire un unicum all'occhio. La mansione protettiva si consuma nella destinazione della pietra a scrigno del "mistero della regalità", perché isola colui che è iniziato a questo mistero dalle impurità e garantisce la trascendenza dell'istituzione. Il marmo diviene memoria del miracolo dell'ipostasi del potere monarchico, che nella morte visualizza e perpetua il sovrano. La pietra al pari delle insegne configura uno strumento di hexis. Assurge a «struttura strutturante» che organizza le pratiche della percezione e quale "struttura strutturata» definisce l'identità sociale nel segno dell'eccellenza. Si riscontra così la mutuazione di strategie cristallizzate a Bisanzio utili ad evidenziare gli spazi concessi alla sovranità posta di fronte all'evento morte.

Lo stesso accade per la monumentalizzazione dello spazio funerario. L'azione dei potenti locali 
si riferisce al precedente costantinopolitano della chiesa-mausoleo e dei suoi materiali di prestigio. Una soluzione che sembra suggestionare le prime esperienze normanne per quel che riguarda la fondazione dei luoghi di riposo della dinastia, quali l'abbazia di Venosa, Cefalù e Monreale. L'ambiziosa progettazione delle strategie locali di auto-rappresentazione dei luoghi della morte deve necessariamente richiedere quella grandezza di intenti che già viene fatta propria dai romano orientali. E se la soluzione delle tombe ducali disposte entro una cappella destinata allo scopo, come quella della Maddalena presso il Duomo di Palermo, trova il precedente autorevole nell'heroon dei Santi Apostoli. Per Monreale il più magniloquente referente architettonico si trova non solo nella chiesa-mausoleo, ma anche nella più ampia concezione di un complesso unico che collega in prossimità la cattedrale al Palazzo, come avviene a Costantinopoli. Tuttavia bisogna ricordare quanto sia importante l'esperienza dei duchi normanni del Nord, che per un certo periodo influenza la scelta dell'allocazione dei sepolcri imperiali e regi presso il coro ed in prossimità dell'altare.

2. RUGGERO I, GLI SPOLIA E LA RIFUNZIONALIZZAZZIONE DELL'ANTICO QUALE GARANZIA D'ECCELLENZA

Un primo esperimento volto all'inserimento del porfido nelle consuetudini architettoniche funerarie dei normanni del Sud si ravvisa nella sepoltura di Ruggero I. Il titolo di Gran Conte ostentato da quest'ultimo non lo legittima all'uso del materiale. Al contempo pare difficile sostenere un'emulatio imperii, perché l'uso del marmo per una trave del baldacchino va liquidato come spolia, specie se accompagnato dal riuso di un sepolcro romano. $\grave{E}$ noto che il riutilizzo dell'antico rappresenta un indice di prestigio, adoperato a fini ideologici (Pensabene, 1990, 5-138). Se questo si somma all'inesistenza dei presupposti in diritto, l'utilizzo del marmo va ascritto come nel caso della Martorana piuttosto ad un "riuso magnificatorio", che "incornicia" in modo fastoso la cassa sepolcrale (Gandolfo, 2006, 202). In entrambi i casi il reimpiego è giustificato dalla particolare bellezza e dalla rarità del materiale, che rivitalizza, rifunzionalizzandolo, l'antico. Il contesto allora sembra fugare ogni rifermento alla tradizione sepolcrale bizantina, longobarda o dei normanni del Nord.

Il riutilizzo dei sarcofagi antichi corrisponde al costume dei papi, che dalla seconda metà del XI sec. ridefiniscono la propria immagine attraverso la riproposizione dell'antico. E se l'imitatio imperii diventa un aspetto fondamentale nella riforma gregoriana, che Ruggero conosce attraverso il sarcofago salernitano di Gregorio VII (Herklotz, 1994, 325), si può osservare che il recupero e la rifunzionalizzazione, una volta correttamente contestualizzati, ne fanno meramente "un monumento autoritario di glorificazione laica» (Deér, 1959, 35) senza ulteriori velleità. L'uso del porfido però non costituisce un «mero reimpiego», ma bensì un "manus facere», laddove i marmi antichi sono rilavorati e riscolpiti (Delli Santi, 2005) ed adattati alla nuova destinazione; ciò a conferma della rifunzionalizzazione. Una soluzione che investe l'alea semantica del segno.

$\mathrm{Da}$ alcune iscrizioni, conosciute solo attraverso citazioni, sappiamo che il marmo viene lavorato da uno scultore romano: Pietro Oderisio, attivo nel sec. XII. Tuttavia il dettaglio prezioso non passa inosservato, rafforza l'alea di significato e il valore sociologico della soluzione sepolcrale e costituisce un precedente per l'architettura di genere. Il marmo rende lo spazio adeguato alla politica di self identity del potere costituito, additando la tomba come venerata.

Il monumento viene smontato a seguito del terremoto del 1659 (Morrone, 1998, n.1, 342-357), come si evince dalle " Notizie degli scavi " del 1882. Il sepolcro viene così ricollocato nel museo archeologico di Napoli. Parte dell'architrave in porfido del baldacchino è rifunzionalizzata presso una Chiesa di Nicotera, laddove funge da gradino d'altare.

Il frammento porfireo presenta tre maschere poste a decoro della stessa architrave. Una ad angolo raffigura un senex caratterizzato da barba, baffi e occhi spalancati. Le altre due protomi invece sono costituite da maschere giovanili, con naso prominente e bocca piccola, mentre le acconciature variano nelle differenti rappresentazioni. Maschere che dimostrano una grande affinità stilistica con i volti del baldacchino sepolcrale palermitano di Federico II (Faedo, 1982).

S'evidenzia ancora la presenza di un altro indizio: il frammento di Nicotera misura la metà dell'architrave del baldacchino di Palermo. Il dettaglio unito alle assonanze stilistiche, non può tuttavia sostenere un intervento diretto di Pietro Oderisio per entrambe le opere, almeno in termini squisitamente cronologici. Probabilmente il monumento del conte Ruggero viene commissionato da 
Adelasia, sua sposa, o al massimo dal figlio Ruggero II; il sarcofago di quest'ultimo invece si ascrive ad una data che oscilla tra il 1145 e l'anno della di lui morte, il 1154. La cronologia che rende impossibile dimostrare sul piano diacronico la presenza di un'unica mano, può piuttosto evocare nel plausibile intervento dello scultore romano l'esponente di una scuola locale, che si confronta con la durissima pietra ed adopera tecniche di lavorazione che spingono a soluzioni assimilabili dal punto di vista stilistico. Il duro marmo obbliga i lapicidi a semplificare i diversi piani con una scarsa attenzione per la profondità. Entrambe le opere poi sono connotate da una generale sommarietà nella resa dei dettagli. Le assonanze morfologiche, più che allo stile, sono certamente dovute alle esigenze tecniche.

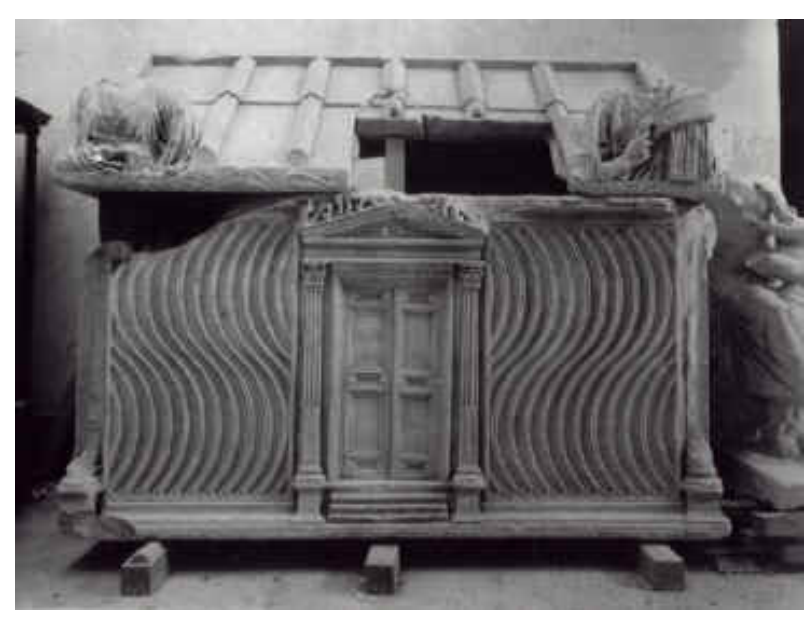

Fig. 1- Sepolcro di Ruggero I, Museo Archeologico, Napoli (immagine da Herklotz, 1995, 324)

3. Pensare una Politica di SelF identity: I Limiti DELL'ESPERIENZA “IMPERIALE” DEI NORMANNI SEGNATA DALL'ALEA DI SENSO DEL PORFIDO

É possibile precisare la significatività sociologica dell'esperienza "imperiale" del Regno di Sicilia, soprattutto attraverso l'abuso del porfido. Questa pietra, che evoca la dignità dell'impero, viene adoperata per i sepolcri dei monarchi romani fino al regno di Marciano, che per ultimo la impiega nelle strategie di auto-rappresentazione dell'istituzione. Sappiamo poi che nel V sec. le cave del Mons Porphyrites, da cui viene estratto il marmo, cessano l'attività, mentre nel sec. X sono oramai esaurite. Ogni utilizzo successivo deve allora essere ascritto ad una politica di reimpiego, volta alla rifunzionalizzazione di spolia o al riutilizzo di giacenze. Un materiale che si presume reperito a Roma e probabilmente sotto forma di colonne da rielaborare.

È alquanto difficile stabilire i veri motivi che spingono gli Altavilla alla scelta del porfido, nonostante si sia tentato in modo efficace di dimostrare che l'origine della svolta autocratica sia da individuare nel rapporto dialettico col papato (Herklotz, 1994, 326). E se i pontefici operano il riuso politicamente orientato di tombe di porfido come quella di S. Elena o della "conca porfirea" collocata innanzi al Pantheon e appartenuta al mausoleo di Adriano, si comprende come i loro vassalli: i re di Sicilia, non possono essere certo da meno.

Eppure non si può trascurare l'intervento dei consiglieri greci che, oltre a fornire tutta una serie di formule di cancelleria che ripropongono un novero di consuetudini bizantine, possono aver suggerito l'uso del porfido. È noto che gran parte dell'ideologia del potere normanno viene fuori dalla dottrina bizantina, ne sfrutta i mezzi cognitivi e le metodologie comunicative. Si percepisce che la panoplia dei segni costantinopolitani del potere è conforme all'aspettativa sociale dei fruitori locali. La sua mutazione appare l'unico modo per opporre un quid pluris ai papi che realizzano l'imitatio imperii. L'imitatio Byzantii sembra uno strumento che ottimizza le possibilità d'affermazione delle politiche di self identity. A maggior ragione se si considera che Ruggero, stando a Giovanni di Salisbury può rappresentarsi, se lo vuole, come imperatore (Ioan. Saresb., Ep. 239, PL II). Il valore sostanziale del porfido esclude poi ogni ipotesi di ricorso a segni alternativi di stato, perché è oggetto non alterabile culturalmente. Esso non tollera né eccezioni e nemmeno infrazioni, in ragione di quel principio della trascendenza delle forme del potere, che il materiale incarna. Ciò è ancor più vero se si considera che l'ostentazione risulta precondizionata ai rigidi limiti dell'esperienza regia, limiti superati dall'appropriazione di segni che del re non sono, come il porfido o la coniazione dell'oro.

In entrambi i casi, come già osservatosi in dottrina, se non si può parlare di "“bizantinismo" dei bizantini», ha a trattarsi sempre di «"bizantinismo" di marca occidentale», che "non per questo cessa di essere influenza bizantina» (Pertusi, 1964, 226). Un'inequivoca bizantinità che permane anche se si mutuano delle soluzioni che i papi oppongono indirettamente ai basileis e direttamente alle loro "creature": gli imperatori d'Occidente. 
La ricostruzione filologica del contesto culturale in cui si realizza l'assunzione del pregiato marmo fra gli essentialia del corredo regio, implica il combinarsi di questo con altri segni. Esso può collocarsi in uno spazio monumentalizzato attraverso un baldacchino, che assolutizza i significanti dello status symbol di fronte all'evento morte. Il sepolcro, quale mobile che contiene il sovrano, diventa un fenomeno architettonico che esalta sul piano visuale il rappresentante dell'istituzione deceduto. Pertanto può essere ritenuto l'insegna più duratura del potere. Il particolare marmo, quale «simbolo di opulenza e di distinzione», si trasforma in una prerogativa che legittima nell'officio chi può ostentarlo. Questo status symbol, quale segno "sensibilissimo" della fenomenologia della regalità, può essere ritenuto un "marchio di eccellenza" ed un "tramite simbolico» (Carile, 2000, 89). L'uso del porfido quale privilegio imperiale si mostra a corollario della «liturgia della porpora» (Cavallo, 1996).

Il baldacchino in porfido assurge a sottoprodotto della bizantinità, che afferma il carattere non derivato del potere normanno in ragione di una nuance con connotazione imperiale (Iannelli, 1982). La sua presenza costituisce un potente indicatore non solo dell'imitatio Byzantii, ma anche di una più generica imitatio imperii, stante l'onnipresenza del baldacchino nelle rappresentazioni del trono dell'Impero d'Occidente. Quest'ulteriore segno garantirebbe l'efficacia comunicativa di certe idee della dottrina del potere locale presso un più ampio pubblico di fruitori. Ma anche in questo caso la sua presenza rimanda ad un "bizantinismo" accolto dagli occidentali.

La presenza delle sei colonne per i baldacchini delle tombe normanne in Palermo, oltre le ovvie ragioni di statica per la copertura di uno spazio più grande, sembra essere piuttosto il frutto di una scelta estetica, volta a costituire un locus rappresentativo. Vi è però difficoltà nel reperire il modello diretto che ha ispirato tale soluzione in carenza di precedenti locali; la tradizione architettonica si limita ad esedre con due o tre colonne che reggono meramente un architrave.

Deér suggerisce un altro precedente che di per sé non ha nulla di imperiale, ma è meramente funzionale: il baldacchino presente nell'antico atrio della basilica di S. Pietro, che copre il Cantharus e la pigna bronzea; quale mera ricezione dell'antico (Deér, 1959, 35).
Uno sparuto modello può essere pure ritrovato nella rara soluzione del baldacchino a capanna presente a decoro di una tomba della metà del sec. XI presso il nartece della basilica romana di S. Lorenzo. Una tomba ragguardevole, ma non certo imperiale.

$\mathrm{Si}$ osserva che il precedente più vicino ideologicamente all'evidenza siciliana consiste però nel sepolcro di Goffredo di Buglione presso la Cappella del Calvario in Gerusalemme, il quale è ora apprezzabile solo attraverso un disegno al f. 103 del Ms. Latino 9233, conservato presso la Libreria Vaticana. Un prototipo consistente in un baldacchino con sei colonne e una copertura cosiddetta a "libretto". Precedente che si lega alla pretesa normanna verso il trono di Gerusalemme e si connette direttamente alla memoria dell'Advocatus Sancti Sepulchri, che re non è mai stato. Eppure l'ideologia regia sebbene smentita dalla titulatio può rientrare in diverso modo. Si considera un diritto che Ruggero vanta per il matrimonio di Adelasia del Vasto, sua madre; costei in seconde nozze diviene regina di Gerusalemme. Il baldacchino, almeno sul piano visuale, può tornare a vantaggio degli Altavilla, quale segno di un più ampio diritto regale. Si vuole spostare l'attenzione dalla prerogativa all'immediatezza del segno, laddove l'imitazione dell'architettura di prestigio non fa altro che potenziare il significato dello status symbol ostentato.

Per comprendere le circostanze in cui attecchisce l'utilizzo del porfido, si deve fare riferimento alla vicende del duomo di Cefalù. Un'aula pensata da Ruggero già dal 1131, che diviene chiesa dinastica dal 1145 quando vi destina la sua tomba monumentale in porfido da collocare nella zona solitamente destinata al clero; una chiesa che costituisce il memoriale della fondazione del Regno (Delogu, 1995, 190).

Anche lo Stato siciliano che si confronta con l'evento morte sente l'esigenza di affermare se stesso e la sua continuità. Per far ciò non può prescindere dalla rituale ostentazione di abbondanza, con cui l'istituzione magnifica se stessa e si racconta. In un momento di cesura si afferma in modo forte, specie attraverso l'ostentazione dei segni del rango, la continuità.

L'abuso del porfido da parte di Ruggero, che esplica il valore sociologico di una pietra assurta a segno del rango, si consuma in un periodo in cui il Regno appare debole sul piano internazionale, stretto fra la pressione dei due imperi e del papato che esprime continue rivendicazioni sul Meridio- 
ne d'Italia, considerandolo feudo del Patrimonio di S. Pietro. L'appropriazione di uno status symbol, posto entro lo spazio di copertura sociale, dimostra che il sepolcro, in ragione del materiale con cui è fatto, aumenta la spendibilità nelle strategie di self identity. Un tale sentire emerge dal diploma di destinazione dell'edificio a mausoleo (Di Gregorio, 1821, 71). Un documento solitamente ritenuto un falso, forse risalente al regno dei due Guglielmi. Tuttavia dal testo non si evince se a quella data i sepolcri forniti di baldacchino fossero già in situ (Valenziano y Valenziano, 1979, 35), fra le braccia del transetto e presso il coro dei canonici. La tomba del sovrano sembra essere collocata a nord, «in cornu Evangelii», mentre l'altro sepolcro, identico e vuoto, forse riservato al successore, forse cenotafio commemorativo, viene allocato "in cornu epistolae» a sud. In Sicilia così continua a rivivere la tradizione tutta normanna dei sepolcri «intra chorum et altare» (La Duca, 2002, 304).

Altrettanto sconosciuto è il luogo di lavorazione dei sepolcri: è probabilmente la cappella palatina palermitana, dove sono abbondanti le tracce di spolia di porfido riutilizzate in un periodo di grande disponibilità del marmo nell'isola. Al contrario in Cefalù è totalmente assente ogni traccia di materiale reimpiegato. Tale assenza fa pesare che in situ si realizza solo il montaggio.

Federico II nella sua ridefinizione delle politiche di self identity nel 1209 trasla i due sepolcri a Palermo. Ciò a tutto scapito di Cefalù e del suo capitolo che vuole ottenere l'inumazione di Ruggero II. Una pretesa già delusa da Guglielmo II, che ha rigettato l'istanza volta alla restituzione del corpo regio. Scelta non indolore, che viene pagata da Federico con la scomunica da parte del vescovo cefaludense. Scomunica che viene revocata solo a seguito della donazione a favore della diocesi del feudo di Coltura, attestata in un atto datato al settembre 1215 presso l'Archivio del Capitolo di Cefalù (Andaloro, 2002, 135).

4 SPUNTI DI RIFLESSIONE SULLA SOCIOLOGIA DEL PORFIDO: SIGNIFICATI E SIGNIFICANTI DI UN SEGNO DEL RANGO

Dalla disamina dei sepolcri emerge una precisa percezione, certamente condivisa da committente e fruitori: è il dettaglio d'eccellenza a fare i re. Senza tale convinzione la scelta del materiale si risolverebbe in un non sense. Si dichiara così la valenza semiotica di un indicatore del rango. L'efficacia sociologica del marmo da prova della predisposizione dei fruitori a recepire lo "spettacolo di Stato", messo in scena nei luoghi del potere che raffrontano l'evento morte.

Le tombe di porfido ri-trasportate a Palermo hanno acceso il dibattito dottrinale. Si può sostenere che siano state adoperate ben due colonne per trarre entrambi i sepolcri, una sicuramente di notevoli dimensioni, con ampia circonferenza ipotetica, ed una forse più piccola. La più grande viene sezionata in due tronconi, forse ad un terzo della circonferenza, per ricavare entrambe le casse. Da quella più piccola, sezionata in linea verticale, invece vengono tratti i coperchi. Ipotesi plausibile in ragione del fatto che la colonna usata per le casse, per quanto grande, non può fornire adeguato materiale di risulta per i coperchi (Gandolfo, 2006, 206).

Si è pensato che in origine Ruggero II ha previsto due sarcofagi sostanzialmente identici, da sistemare in maniera simmetrica davanti al presbiterio. Cosa che non spiega la maggiore ricchezza del sarcofago occupato poi da Federico II rispetto a quello di Enrico VI. Ciò ha suscitato una sparuta ipotesi: i sarcofagi cefaludensi dovrebbero essere identificati con quelli quasi identici, adoperati per l'inumazione di Costanza d'Altavilla ed Enrico VI; una tesi smentita dai fatti.

Barolo sostiene che solo la cassa del sarcofago di Federico II possa appartenere all'evidenza cefaludense. Il più ricco apparato iconografico delle mensole zoomorfe e del coperchio con spioventi decorati da clipei potrebbe essere ricondotto ad un bildprogramm voluto da Federico, che rimodula l'evidenza (Barolo, 2002, 4). Una tesi che appare plausibile, se si considera che la ri-funzionalizzazione ribadisce il diritto del ramo svevo al titolo di re di Sicilia. L'innesto svevo nella casa degli Altavilla si consuma anche nell'appropriazione dei segni di status esclusivi, come la tomba di porfido. La scelta, che va incontro all'aspettativa del pubblico, tiene conto di qualcosa di più. La monarchia non può tradire se stessa e deve fornire ai fruitori quel novero di segni che la magnificano, la raccontano e ne rendono migliore la spendibilità. Sono i segni di status che ne aumentano la comprensibilità, almeno degli aspetti esteriori. Ormai la significatività del segno transita all'oggetto che da esso è ricavato. L'oggetto diviene segnacolo del rango auto-legittimante e permette di riaffermare continuità e legittimità della posizione del committente (Vergara Caffarelli, 2002, 317).

Il sarcofago occupato da Federico II presenta la forma di arca. Il coperchio a spiovente appare de- 
corato su entrambi i lati con una sequenza di tre clipei. Il posto d'onore è riservato su un lato al Cristo Pantocratore e sull'altro all'Odeghitria con bambino, mentre nei restanti clipei si dispongono i simboli degli evangelisti a conferire un carattere escatologico e teofanico al bildprogramm. Ai lati del coperchio viene ancora rappresentata la bügelkrone, col doppio arco ad ogiva; la stessa corona che si colloca all'apice dello stemma personale di Federico. Insegna maschile che compare su un capitello con la scena del dono alla Vergine presso il duomo di Monreale, ma anche nel f. 26 del "Liber ad honorem Augusti"; l'insegna potrebbe alludere alla corona propria del Re di Sicilia. La cassa del sepolcro, di forma concava, presenta un elegante bordo rifinito con fregio vegetale, costituito da foglie d'acanto. I lati della cassa sono ornati uno con una croce greca patente, mentre l'altro versante presenta un battente con testa leonina.

Le mensole zoomorfe mostrano dei leoni accovacciati, con le code intrecciate ed annodate al lato. Il volto dell'animale appare stilizzato e sembra ricordare una maschera, mentre la criniera a riccioli evoca pure il mantello di Ruggero II. Il leone, quale animale totem, assume la funzione di guardiano dei sepolcri. Fra le zampe dei leoni vengono collocate diverse figure in genere antropomorfe o zoomorfe, di cui si citano ben due che colpiscono l'immaginario. Un leone trattiene e schiaccia un uomo, il cui volto imberbe è caratterizzato da un'espressione di chiara terribilità, quale cachet della scultura medievale sepolcrale. Un tema quello della violenza, quale espressione antropologica della forza ritualmente ostentata dal sovrano, che si ripete nell'altro animale che trattiene un capretto. Un altro leone ha collocato fra le zampe una strana figura antropomorfa, consistente in un uomo seduto capovolto ed accovacciato con le mani poste sulle ginocchia, che emerge dal corpo dell'animale. Il volto di questo personaggio si presenta caratterizzato da baffi e da occhi spalancati; tratti resi con gusto naif. Un personaggio identificato persino con un ritratto di Federico.

Occorre riflettere ancora sulla funzione gnoseologica del monumento, quale condensato dell'immaginario di genere. Un corredo tematico e simbolico a finalità narrativa che l'uomo medievale ha ben presente. Il sepolcro costituisce una descrizione retorica dell'istituzione, che si muove fra il cristianesimo e quel paganesimo che il costume funerario cristiano accoglie. Si presume che il pubblico, almeno l'èlite, sia in grado di percepire gli inputs che la dottrina del potere suggerisce. Si può parlare allora di una coscienza del valore narrativo del monumento da parte dei fruitori.

Il sepolcro è poi collocato sotto un baldacchino di porfido rosso sorretto da colonne, che viene probabilmente danneggiato durante il trasporto a Palermo ed integrato con inserti in granito. La durezza del materiale impone ai lapicidi di ripiegarsi sulle foglie d'acqua per i capitelli che interpretano lo stile corinzio. La morfologia lanceolata del vegetale non richiede particolare attenzione per i dettagli interni. Fra questi spicca un capitello in granito, che presenta motivi zoomorfi costituiti da aquile e grifoni ed integra una componente danneggiata.

L'architrave è invece ornata da maschere antropomorfe. In particolare il volto barbuto mostra estreme affinità stilistiche con i due visi alla Dumbarton Oaks Collection, tanto che Deér ha ipotizzato l'unicità della mano esecutiva. La sinteticità dei tratti, quale soluzione di compromesso con la durezza del materiale, permette appena di indicare l'età dei rappresentati.

Le capriate sempre in porfido sono ornate da due colonnette tortili per lato e sorreggono una copertura a libretto in lastre di granito. Quest'integrazione dimostra che al momento della ridefinizione è impossibile anche per un imperatore poter reperire sul mercato del porfido.

Il sepolcro, quale segno di status, sembra suscitare una precisa suggestione, che evoca il locus già esplorato da Kantorowicz del doppio corpo del sovrano (Kantorowicz, 1989). Questi si presenta al contempo vivo, seppur il suo corpo riposa nel sepolcro, giacché vive ancora come corpo "pubblico". Dalla sede d'eccellenza del porfido il trapassato ostenta la pienezza del suo potere nelle forme delle onde mnemoniche; un significato che viene instillato attraverso specifici simboli della dignità propri dell'ambito funerario e che anche l'uomo medio sa leggere. L'attenzione al dettaglio prezioso vuole comunicare quei valori sociologici altamente condivisi e posti alla base delle forme di rappresentazione. Il segno ottimizza la trasmissione di informazioni ed obbliga il fruitore alle comportamentalità connesse al timor reverentiae del rango. Una tendenza che si rafforza di fronte all'evento morte, allorquando dalla persona del sovrano defunto non ci si può più aspettare niente. I segni d'eccellenza hanno così il compito di rimarcane l'autorevolezza. La cura nella costruzione di un apparato di segni "forti" ed 
adeguati al sepolcro del sovrano ha funzione performante, ottimizza percezione e gradimento del segnacolo.

Eppure la ricca decorazione dello spiovente e delle mensole del sepolcro di Federico II potrebbero additarsi piuttosto alla commissione del Balio Manfredi e di Berardo di Castaca, arcivescovo di Palermo. Costoro avrebbero incaricato Jacopo Tedesco di Lapo di realizzare i rilievi del coperchio e le due mensole zoomorfe (Ramata, 1955).

Tuttavia sulla scia di Deér anche Gandolfo può sostenere una datazione precoce del sepolcro di $\mathrm{Fe}-$ derico II (Deér, 1959, 50-54; Gandolfo, 2006, 207). Ciò per ragioni stilistiche. Il duro porfido deve essere lavorato da quelle maestranze specializzate gravitanti attorno alla corte, le stesse che hanno scolpito il candelabro pasquale della cappella palatina. Le affinità stilistiche permettono di accogliere una datazione intorno al 1154, anno della morte di Ruggero, o fors'anche di qualche anno dopo. Sono lapalissiani i legami fra i leoni alla base del candelabro e quelli delle mensole. Si ritrovano le medesime zampe con giunture nodose e artigli allungati. In entrambi le articolazioni appaiono rigide e le criniere vengono delineate da ciocche terminanti ad uncino. Altrettanta affinità si riscontra fra il mo-

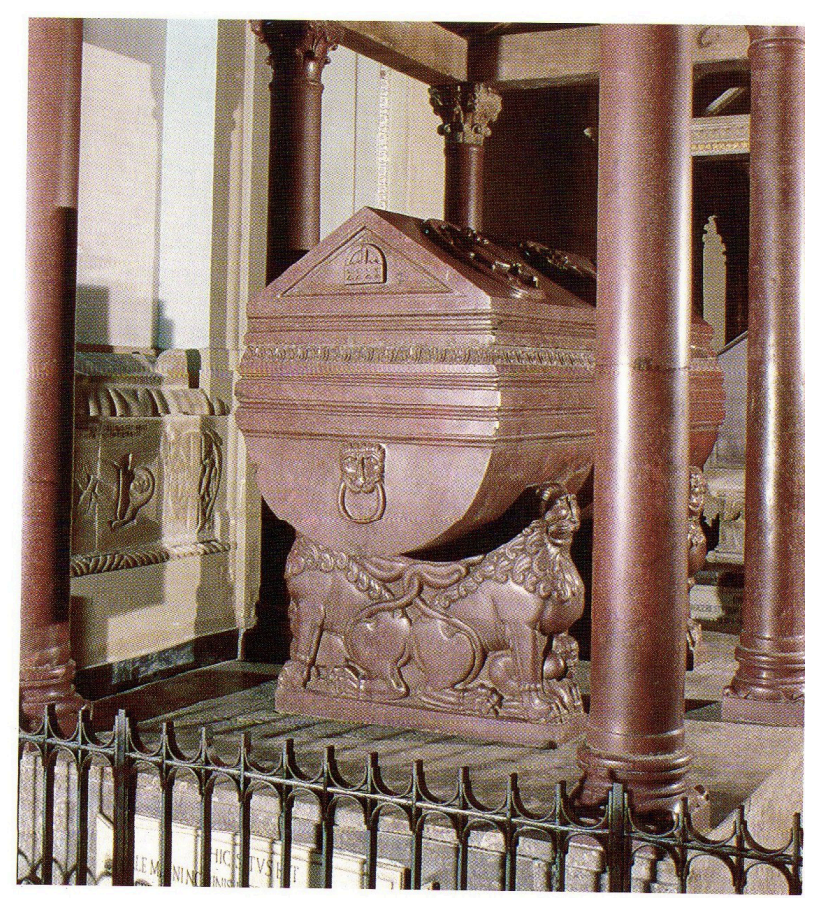

Fig. 2- Sepolcro di Federico II, cattedrale, Palermo (immagine da Gandolfo, 2006, 206, fig. 8). dellato del volto del Cristo del candelabro e quelli del Cristo e della Vergine del sepolcro. Anche i simboli del tetramorfo appaiono assimilabili alle bestie che si dispiegano lungo il candelabro. Analogie, che nell'indimostrabilità dell'opera dei singoli autori, si spiegano nell'ambito di una scuola palatina specializzata nelle tecniche di lavorazione del porfido. La mancanza di virtuosismo nella traduzione plastica e l'assenza della resa della profondità fra i piani sono un marchio tecnico della scuola. Questi sono null'altro che espedienti per assecondare la durezza del porfido, dato che le tecnologie antiche di lavorazione sono andate perse con l'esaurimento delle cave egiziane. Una durezza che costringe a soluzioni di sintesi.

Ben più semplice è il motivo del sarcofago occupato da Enrico VI, il cui programma ornamentale rimanda però ad un precedente stilistico autorevolissimo: la conca porfirea di Adriano, ora nella cappella Corsini dove accoglie le spoglie di Clemente XII. La scelta mostra una preferenza per "l'antico", specie in un momento in cui i vezzi della moda suggeriscono di optare per la più moderna soluzione del giacente; il ricorso ad una tradizione formale che si può definire "classica", unita all'impiego dello status symbol, costituisce un consapevole strumento di legittimazione.

Il sepolcro anche in questo caso è caratterizzato da un tetto a spiovente, ma privo di decori. Su uno dei lati corti compare un motivo floreale, assimilabile ad una rosetta ad otto petali, mentre nell'altro si iscrive una corona che sempre Deér definisce prettamente femminile. Nella generale sobrietà dell'evidenza, non si può però ignorare la presenza del bordo profilato con ovuli, che costituisce una significante citazione del bildprogramm imperiale. In questo caso il lato breve del sarcofago appare ornato da un motivo vegetale, forse una foglia di edera, inserita in una corona. La cassa a sua volta è poggiata al di sopra di una doppia mensola modanata.

L'evidenza sul versante plastico vuole rappresentare in modalità molto semplificate il modello romano e le sue sinuose linearità. L'opera dei lapicidi locali è indirizzata a trovare soluzioni per riportare il modello. Tuttavia la notevole riduzione in spessore dei corpi scanalati e delle volute dei sostegni si inserisce in un percorso che segna la riscoperta della complicata tecnica per lavorare il porfido.

Il baldacchino di porfido, che è rimasto integro, enfatizza lo status imperiale attraverso la profu- 
sione del materiale dal colore significante. I capitelli sono anche qui piuttosto stilizzati, il motivo del decoro perde la grazia dell'acanto per aderire nuovamente alla morfologia delle foglie lanceolate. Le architravi presentano una cornice lineare ed un timpano altrettanto sobrio, che considera l'unico fasto del porfido. Le capriate mostrano nel mezzo una semplice colonnina, mentre le lastre che compongono lo spiovente del tetto sono ancora una volta prive di decorazioni.

Per meglio comprendere l'ulteriore valore dei sepolcri, quale medio legittimante in chiave dinastica, deve piuttosto stigmatizzarsi la volontà di Manfredi di celebrare la propria incoronazione nel duomo di Palermo, innanzi alle tombe degli avi naturali. Ciò al fine di garantire anche a medio visuale la continuità tra il suo officio e quello ricoperto dal padre biologico; in tal modo si afferma un diritto che Manfredi Lanza vanta ius sanguinis.

Malaspina ha a sottolineare come Manfredi, nella posizione di figlio legittimato, opta per una cerimonia officiata «in Ecclesia tandem Panormitana, quam Regum Siciliae porphyrea et anabastrica monumenta materia et arte pretiosa decorant» (Malaspina, Rer. Sic. Lib., I, 7, II), per supplire a quel che manca al proprio status. Traspare una certa attenzione verso l'herrschaftssymbolik, che rispecchia la mentalità simbolica dell'uomo medievale. Emerge un peculiare interesse verso questi segni sensibili del potere e verso il loro valore ontologico.

Secondo Vergara Caffarelli la scelta potrebbe essere connessa all'ipotesi, ovviamente tutta da verificarsi, di un Manfredi esecutore del legato testamentario pertinente alla composizione delle tombe imperiali e regie (Vergara Caffarelli, 2002, 317). Il probabile ruolo diviene un efficace mezzo di propaganda in chiave d'auto-affermazione tutta dinastica. Manfredi, probabile esecutore testamentario di Federico II insieme all'arcivescovo di Palermo Berardo de Castaca, ha interesse a magnificare l'opera di pietà verso la tomba paterna nel momento d'accesso al regno. A maggior ragione se in linea di successione è pretermesso a Corrado, erede universale, ai di lui figli, a re Enrico ed eventuale prole (Renda, 2012, $193 \mathrm{sgg}$ ). Una ricerca di legittimazione che utilizza i sarcofagi di porfido come "collante" simbolico fra le proprie aspirazioni, la monarchia del padre e quella del bisnonno. Il porfido sul piano visivo, ma anche ideologico, può permettere di superare lo stallo dovuto allo stato di figlio naturale (Molina López, 2014, 59).
Deve ancora significarsi che Costanza, a mezzo del suo testamento datato al 25 dicembre del 1198, palesa il desiderio d'essere seppellita presso le tombe del padre Ruggero II e del marito Enrico VI, ubicate nella Cattedrale di Palermo. Per questa si progetta, forse su commissione di Federico, un sepolcro in porfido che rappresenta non solo l'ultima grande impresa degli opifici palatini, ma anche il frutto di sapienti orchestrazioni nei lavori d'intaglio per far fruttare il poco porfido disponibile. Il modello da seguire è quello romano, nella versione semplificata locale, quale imitazione dell'imitazione. Proprio il modello pedissequo ha tratto in inganno certa dottrina e lo stesso Deér, che ha immaginato per Costanza il recupero di una delle due tombe di Cefalù. Eppure alcuni elementi sono sufficienti a smentire tale asserzione. Uno è ideologico, legato alla concezione del monumento quale palese imitazione. L'altro concerne la qualità del materiale utilizzato, che documenta la poca disponibilità al momento della produzione. I criteri esecutivi dimostrano che in quel momento non si ha a disposizione un'intera colonna, ma solo una sezione di essa, forse pari ad un terzo. I lapicidi riescono a ricavare da un pezzo unico il fondo della cassa sul cui lato corto iscrivono una ghirlanda contenente una croce greca patente. Procedono al montaggio del coperchio, dei lati e

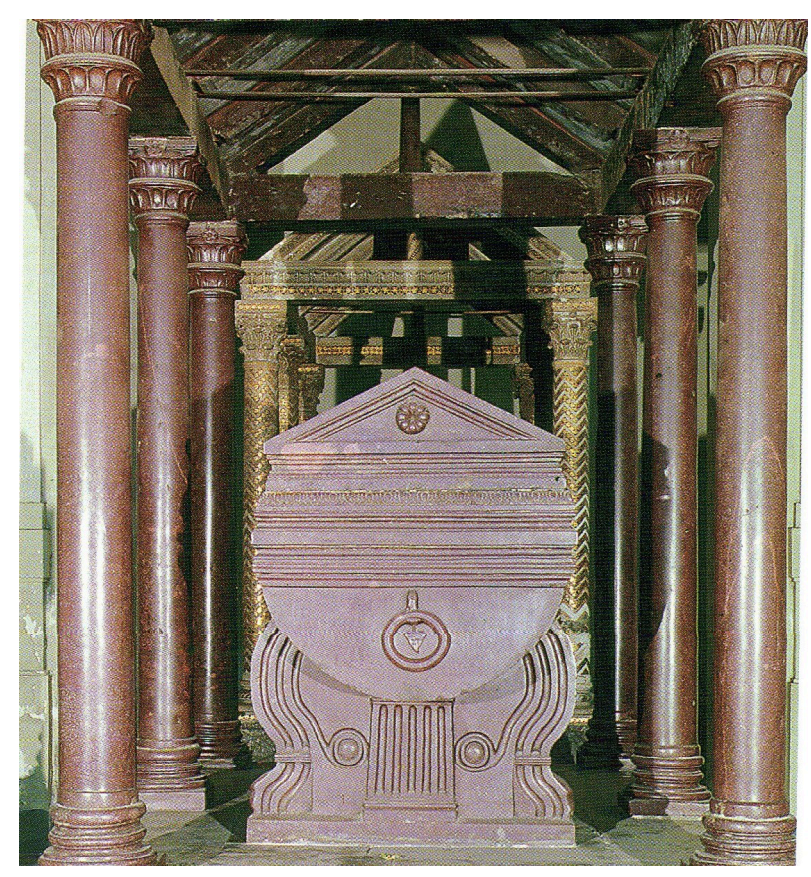

Fig. 3- Sepolcro di Enrico VI, cattedrale, Palermo (immagine da Gandolfo, 2006, 206, fig. 9). 
delle mensole traendoli da lastre frutto di un'opportuna sezione del materiale restante. Il coperchio in particolare risulta frutto del montaggio di diversi pezzi e come nelle tombe alla cappuccina lo spiovente è composto da lastre inclinate ed appoggiate l'una all'altra. Una delle cuspidi poi accoglie il rilievo di una bügelkrone, attributo maschile per Deér, che ha dato adito in dottrina alla querelle su una errata composizione dei corpi di Costanza ed Enrico dopo qualche frettolosa ispezione (Deér, 1959). Allo stesso modo le mensole sono costituite da due lastre sovrapposte e di spessore addirittura dimezzato rispetto al sepolcro erriciano. Di conseguenza la qualità e la quantità della decorazione va rimodulata in proporzione alla poca pietra posseduta. Un fattore che spinge ad una modesta interpretazione del prototipo, specie nelle mensole caratterizzate da poco profonde scanalature e dalla resa sommaria del fregio ad ovuli.

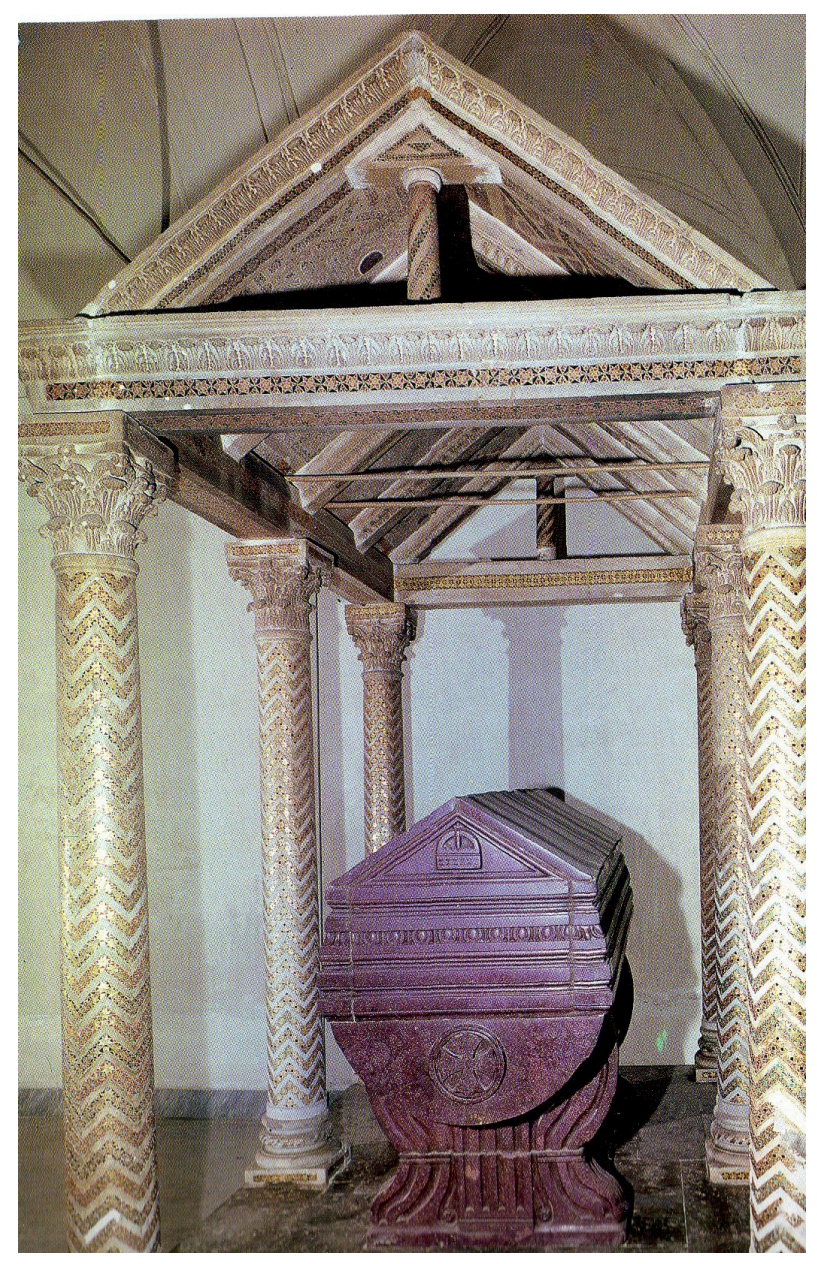

Fig. 4- Sepolcro di Costanza d'Altavilla, cattedrale, Palermo (immagine da Gandolfo, 2006, 213, fig. 20).
L'indisponibilità del porfido costringe a adoperare per il baldacchino a sei colonne il marmo bianco e non il materiale che conviene ad un'imperatrice, decorato però in mosaico con motivi a zig-zag. I capitelli presentano un gusto classicheggiante in ragione della voluttà del fogliame d'acanto posto a decoro; vezzi che il più malleabile marmo permette. Anche in questo caso sia la doppia capriata, sia le architravi, sia il timpano possono essere decorati con un motivo continuo a foglie d'acanto; compare anche un'elegante colonnina a tortiglione. Il tetto a libretto è connotato poi da decorazioni cosmatesche a motivo geometrico, che aumentano l'incisività della monumentalizzazione della sede, nonostante la loro banalità rispetto alla destinazione.

C’è persino chi ha ipotizzato il riuso di una tomba già esistente, a destinazione maschile, data la corona. Forse quella sita nella cappella palatina e destinata a Guglielmo I o quella occupata da Tancredi, da cui il marito Enrico VI ha fatto sottrarre le insegne regali. Indi per cui, se eseguita per $\mathrm{Gu}-$ glielmo I, si dovrebbe collocare ad una data ante quem che si fissa al 1183. Se la si vuole identificare con quella riservata a Guglielmo II o persino a Tancredi, la si deve collocare invece entro una data post quem superiore al 1194. Tuttavia in assenza di maggiori indicazioni tali ipotesi appaiono indimostrabili, anche perché il tentativo d'imitazione, nella sua modestia, non offre dettagli utili a delineare una cronologia specifica.

5. Auto-rappresentanzione E falsificaZione di UN CODICE. L'ESPERIENZA DEL POTERE LETTA ATTRAVERSO LA TOMBA DI RUGGERO II

L'abuso del porfido per la tomba di un re, contro ogni prerogativa di diritto, pone in essere la falsificazione di un codice e investe la percezione dell'istituzione regia per quel che è. Ma il porfido in Sicilia è ormai pertinenza simbolica e giuridica della casa reale.

Innanzitutto devono spiegarsi le cause che permettono alla cattedrale di Palermo di accogliere la tomba reale ed all'arcivescovo Walter di Mill di imporsi sull'esecuzione dei legati testamentari di Ruggero. Alla morte del re, il 26 febbraio 1154, la chiesa dinastica di Cefalù non è ancora consacrata ed urge una collocazione per la salma. Il vescovo si assicura in questo periodo la sepoltura regia che fornisce prestigio alla propria sede, tanto da assumere un ruolo primaziale sull'intero Regno. Privando la cattedrale cefaludense delle sue funzioni, si da adito 
ad una lunga diatriba che si trascina fino al regno di Federico II.

Questo vescovo prevede per il sovrano un'urna provvisoria, delle cui fattezze si sa poco o nulla, provvisoria perché sembra essere utilizzata fino al 1170 (Perricone, 1916, 16). Dopo quella data l'arcivescovo Gualtiero commissiona la riedificazione della cattedrale. Durante questo periodo si ignora l'eventuale collocazione delle spoglie. Si può però ipotizzare che nel 1187, il sepolcro si trovasse già nel transetto meridionale, perché un'iscrizione del 1185 sita nell'abside ci informa che il corpo è già ultimato. A quella data la salma viene deposta in un'arca dalle forme lineari, che ricorda un reliquiario limosino, sobrio e quasi minimale, in cui l'unico fasto è dato dal porfido con cui viene costruito.

Per la tomba di Ruggero II l'ambizioso vescovo committente si scontra col problema della carenza del porfido, che viene risolto con abili soluzioni. Il porfido allo stato dei fatti può divenire appannaggio di un privato, seppur sia il potente vescovo palermitano, solo in ragione della sepoltura di un re. L'uso del porfido non solo deve risarcire la volontà testamentaria di Ruggero già disattesa, ma deve opporsi al capitolo cefaludense che in virtù del sepolcro reclama l'esecuzione testamentaria; deve pure contrapporsi a Monreale, divenuta sotto Guglielmo II il pantheon regio. Tale opzione ribadisce il significato sociologico di un segno essenziale nelle strategie di auto-rappresentazione del sovrano defunto. L'urna è creata dalla mera connessione di lastre di porfido, tratte dalla sezione di un blocco non troppo grande e forse di risulta. La soluzione appare di compromesso, supplisce alle carenze di porfido e soprattutto alla relativa impossibilità per un privato d'accedere ad un bene appannaggio della casa reale. Le tecniche di montaggio e collazione suggeriscono la difficoltà nel reperire blocchi di porfido di una dimensione adeguata da rilavorare. Gli ingenti costi che il vescovo deve sobbarcarsi per reperire il materiale obbligano poi ad optare per un progetto diverso da quello adoperato per le tombe di Cefalù.

L'unica velleità plastica che viene concessa è quella dei telamoni in marmo bianco, che fungendo da mensole, non solo sostengono la cassa, ma creano un gradevole contrasto cromatico. I telamoni sembrano impegnati nello sforzo immane del sollevare l'urna; sforzo che viene esteriorizzato dalla terribile espressione dei volti di questi. Una mimica che richiama un locus adoperato per la cattedra di Elia nella basilica nicoliana di Bari. Come nell'esemplare barese spicca la terribilità dei volti degli uomini che sostengono il segno del potere. I quattro sono ascrivibili morfologicamente alle etnie su cui si estende il Regno di Sicilia nel suo massimo splendore. Fra questi compaiono i tratti fisici del tipo camusso, quelli ascrivibili alla traduzione plastica del tipo bizantino, ad adombrare una sommessa aspirazione imperiale. Nonché quelli identificabili con i tratti di un arabo e infine quelli dell'uomo occidentale.

Deve segnalarsi che la prossemica dell' «affondo» che caratterizza i telamoni, unita alla gestualità delle braccia solitamente rivolte all'indietro, appartiene ad una formula rappresentativa bizantina (Demus, 2008, 59). Formula che viene qui modificata ed adeguata al fine di rendere più icastico lo sforzo nel sollevare il peso impostogli. L'erronea collocazione dei telamoni, successivamente ai lavori del Fuga presso le estremità del sarcofago, ha costretto le sculture a sopportare un peso maggiore di quello per cui sono state concepite, cosa che ne causa il progressivo sgretolamento. Tale ipotesi viene confermata sul piano filologico dai disegni di Daniele (Daniele, 1784, 147 sgg). I telamoni a loro volta poggiano su un basamento rivestito in lumachella grigia, proveniente da Cefalù.

Il baldacchino in marmo dimostra l'effettiva carenza del porfido e l'uso di strategie rappresentative alternative. Si ricorre allo splendore delle tessere d'oro a mosaico che ornano le sei colonne del fastigium; i motivi cosmateschi suggeriscono nella loro convenzionalità il dispendio di risorse tipico dello stile di Ruggero. Quattro colonne presentano un disegno a zig-zag policromo, mentre le restanti due sono decorate con motivi a rombo. Esse sono a loro volta coronate da capitelli corinzi che interpretano il gusto classico. Questi reggono poi una fastosa architrave a motivo vegetale. La copertura a falde del fastigium, resa con quattro pannelli mosaicati a motivo geometrico, viene sostenuta da capriate in marmo, che poggiano su un'elegante colonnina centrale.

Il sepolcro di Ruggero vive alterne vicissitudini, nel 1781 la parte posteriore del corpo dell'arca viene esportata, per essere sostituita provvisoriamente da una tavola di legno che lo chiude pietosamente. Il pezzo di porfido viene trasportato a Torino su ordine di Vittorio Amedeo di Savoia, divenuto nel frattempo principe di Sicilia (Daniele, 1784, 115). La lastra ricompare solo nel 1861 per essere ricollocata sull'arca. 


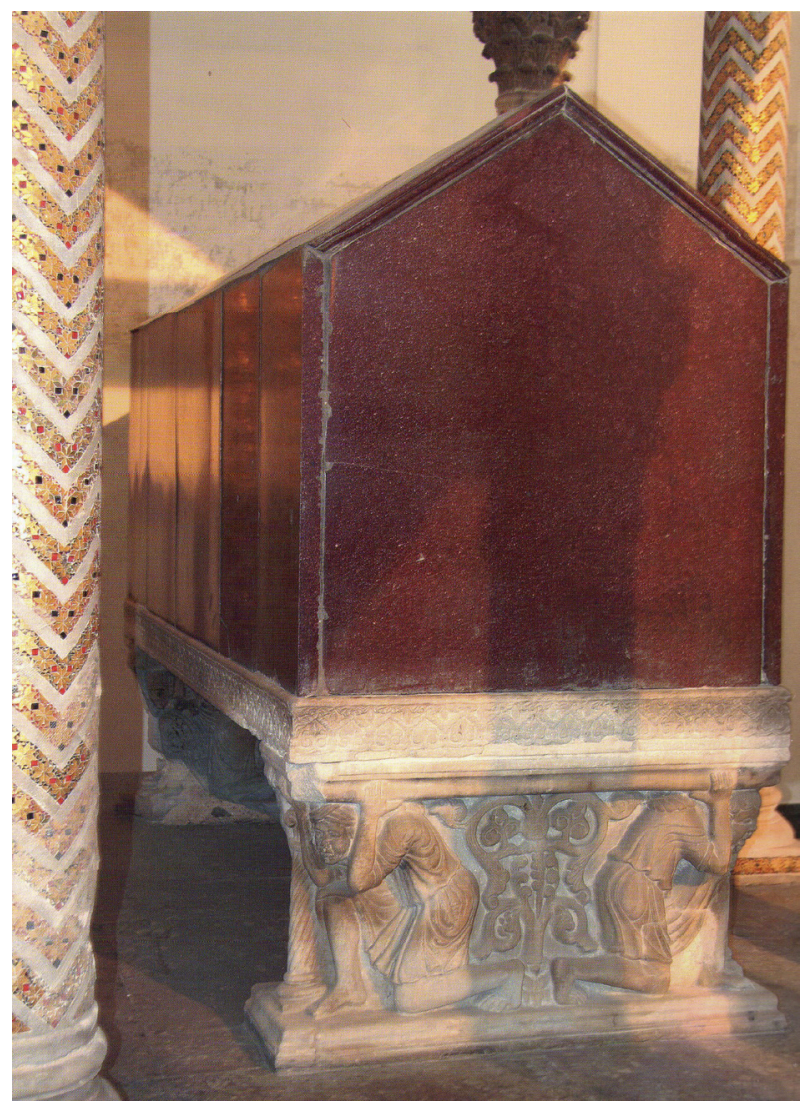

Fig. 5- Sepolcro di Ruggero II, cattedrale, Palermo (immagine da Gandolfo, 2006, 212, fig. 19).

6. La PARABOla VITALE DEL «CIMITERO DEI RE»: UNA VICENDA COMPLESSA A MARGINE DELLA STORIA DELLA CATTEDRALE PALERMITANA

Le vicende della sede del potere normanno che si confronta con l'evento morte si intrecciano con il ciclo vitale della cattedrale di Palermo: qui si accolgono le salme dei duchi di Sicilia e delle regine presso la cappella di Santa Maria Maddalena (Daniele, 1784, 115). Questa viene distrutta da Gualtiero d'Ofhamill per poter proseguire i lavori di rimodernamento del duomo. Tuttavia per far ciò si ha bisogno del benestare del regnate, Guglielmo II, a cui si indirizza apposita petizione. La mancata menzione di Ruggero lascia credere che sia stato sepolto altrove. Durante questi lavori il vescovo definisce la sepoltura di Ruggero, oberandosi fors'anche degli ingenti costi del porfido. Forse perché Guglielmo II è tutto preso dalla realizzazione di Monreale e difficilmente può seguire il progetto per il fondatore del Regno.

Nel lungo periodo fra la fine del sec. XII e la prima metà del sec. XIII si realizzano plurimi lavori di risistemazione dell'aula liturgica. In questo lasso di tempo i sepolcri, che costituiscono il "Cimitero dei re», trovano la propria sistemazione temporanea.

Ad una data presunta fra il 1184 e il 1187 si sceglie per i sepolcri una collocazione a meridione ed a sinistra dell'altare. Quest'ubicazione può attribuirsi all'arcivescovo di Palermo, Gualterio, che ristruttura la cattedrale (Barolo, 2000, 8).

Federico II, nel lasso di tempo intercorrente tra il 1209 ed il 1215, ordina la rimozione e il trasporto a Palermo dei due sarcofagi collocati da Ruggero II nel Duomo di Cefalù. Sembra che Federico abbia previsto la ricollocazione del sarcofago del fondatore del Regno «in cornu Evangelii», seguendo la planimetria desiderata dallo stesso Ruggero II per Cefalù (Valenziano, 1979, $35 \mathrm{sgg}$ ). Specularmente l'altra evidenza viene destinata a sé e collocata «in cornu epistulae». La posizione secondaria sembra inserire Federico in una linea di continuità di governo tutto familiare. Una continuità in linea femminile, che viene ribadita dalla presenza della madre Costanza (Vergara Caffarelli, 2002, 317). L'espediente fa ipotizzare una simmetria nella collocazione delle urne, che deve necessariamente opporre a Costanza la tomba del marito Enrico VI.

Una sistemazione che viene forse approntata solo sotto Manfredi ed a seguito di circa un cinquantennio di alterne vicende. A riguardo dell'allocazione più precise informazioni giungono attraverso un disegno a penna acquerellata proveniente dal "Teatro Geografico Antiguo y Moderno del Reyno de Sicilia", un manoscritto datato all'anno 1686, ora alla Biblioteca Nacional de Madrid (La Duca, 2002, 308, nota 34), che li mostra presso la cosiddetta Cappella del SS. Sacramento.

Il monumento di Ruggero II viene collocato a destra insieme a quello di Costanza. Le tombe di Federico ed Enrico si pongono a sinistra. Entrambe le coppie di urne vengono sostenute da due basamenti in marmo bianco fornito di tre gradini speculari. Diversamente non si comprende bene se per Ruggero e Costanza il baldacchino sia unico e contrassegnato da sei colonne come certa letteratura riferisce o se ve ne siano due, uno per sepolcro, come il disegno del "Teatro Geografico Antiguo y Moderno del Reyno de Sicilia" sembra far credere. Maggiori dettagli li aggiunge Mongitore, che osserva la presenza di cancellate in ferro e di preziosi vela in seta, tenuti da anelli mobili fissati ad aste di metallo, che si ineriscono fra i capitelli del fastigium (Mongitore, 1973, Qq. E. 3., 415). 
Durante i lavori del 1781 in cui è deciso l'imbarocchimento dell'aula, le tombe vengono collocate presso la campata della navata laterale destra in propiscenza dell'ingresso meridionale. Ultimo passaggio di una travagliata storia prima della collocazione attuale presso la prima cappella della navata occidentale su progetto dell'architetto Ferdinando Fuga.

L'attuale cappella dominata da decori in stucco e da un teschio incoronato fra festoni, colloca i sarcofagi a Nord e suggestivamente enfatizza la provenienza di Federico II.

La ricerca ha fatto luce su un'altra interessante vicenda che ha coinvolto questi sepolcri nel sec. XVI, allorché si è progettato il trasferimento delle urne di porfido in Spagna. Già nel 1954 Catalano ha reperito presso l'Archivio di Simancas una lettera fornita di un disegno del sepolcro di Federico II, che non solo informa sullo stato di conservazione del sarcofago al 1577, ma conferma che il coperchio al 1781 si trova in una posizione diversa rispetto all'originaria.

Il documento consiste in una lettera a firma del Capitano Diego Ortiz de Urizar. A tramite di questa si propone al Segretario di Stato, don Antonio Pérez, il trasferimento dei sepolcri della Cattedrale di Palermo presso il monastero di San Lorenzo de El Escorial. I sovrani normanno-svevi verranno così tumulati in altri sarcofagi di marmo da costruire all'uopo.
A tale progetto la risposta di Filippo II giunge chiara:

He visto esto pero no quiero quitarlo de adonde está, que no digan que por componer un Santo descompongo otro, y todavía allá lo sentirán. (cit. in Catalano, 1965/66, 184).

Una simile risposta potrebbe essere causata non solo dalla lungimiranza politica, volta alla salvaguardia del favore dell'opinione pubblica locale, ma sembrerebbe piuttosto dettata dallo scarso successo di un precedente intervento sulle tombe regie e dalle proteste seguite alla risistemazione nel 1557 della cosiddetta Porta Romana in Capua, voluta da Federico. Il rischio di intraprendere un'operazione poco popolare ha fatto desistere da ogni intento.

Se ne deduce che i sepolcri al pari degli altri segni del potere, costituiscono realtà tangibili e sono da considerarsi effettivi segnacoli della dignità regia siciliana e ancor più imperiale, segni che non possono non incidere l'imaginerie di quei potenti che hanno ad occupare il trono di Sicilia. Costoro hanno interesse a dimostrare la continuità di governo attraverso l'uso di questi segni legittimanti. Segni che suscitano il desiderio di emulazione, quando è possibile, come accade ad Alfonso X il Saggio che si riserva un sepolcro in porfido, mentre Filippo II si accontenta del marmo verde.

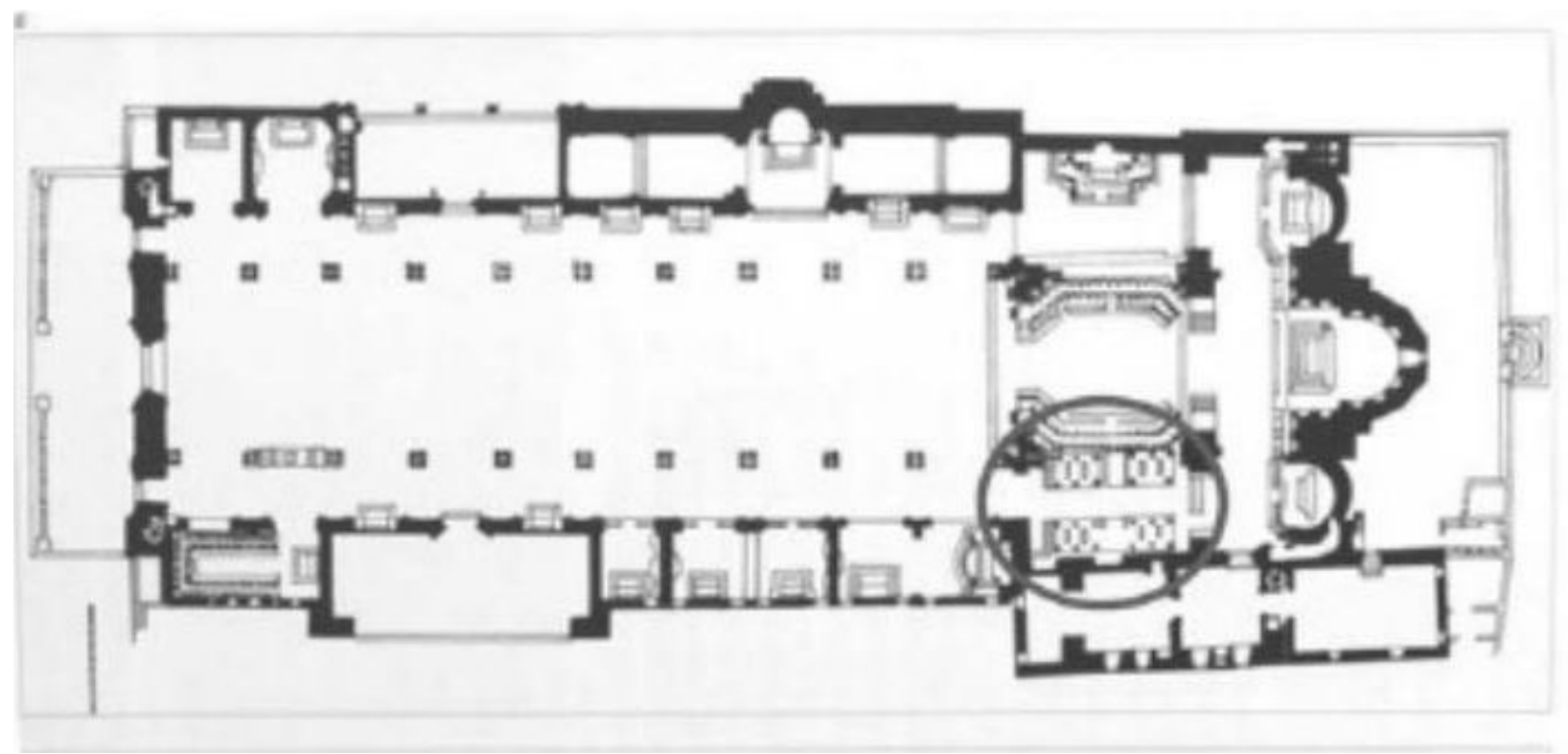

Fig. 6- Planimetria della Cattedrale di Palermo nel periodo normanno (immagine da Andaloro, 2002, 141). 


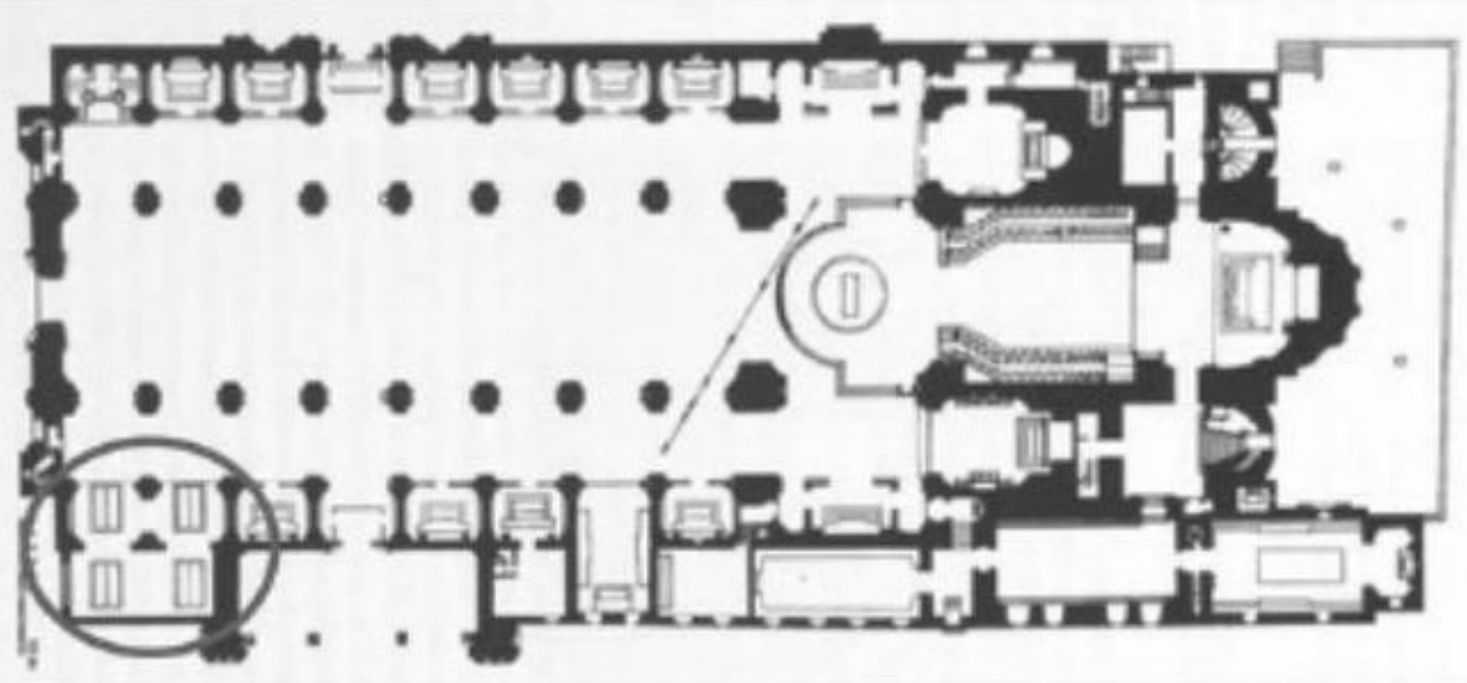

Fig. 7- Planimetria della Cattedrale di Palermo dopo il 1781 (immagine da Andaloro, 2002, 141).

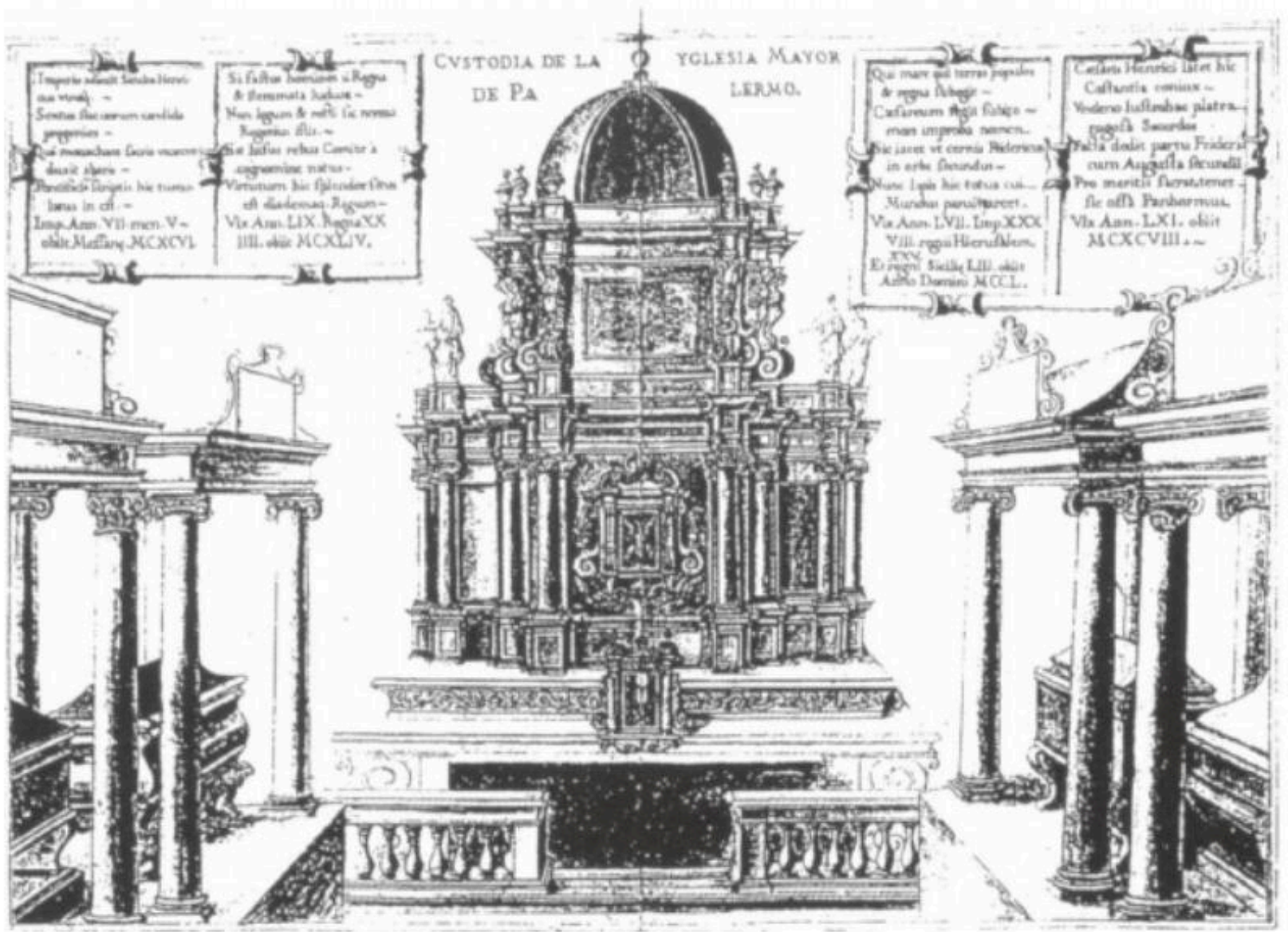

Fig. 8- Disegno seicentesco con la rappresentazione del «Cimitero dei Re» (immagine da La Duca, 2002, 308). 


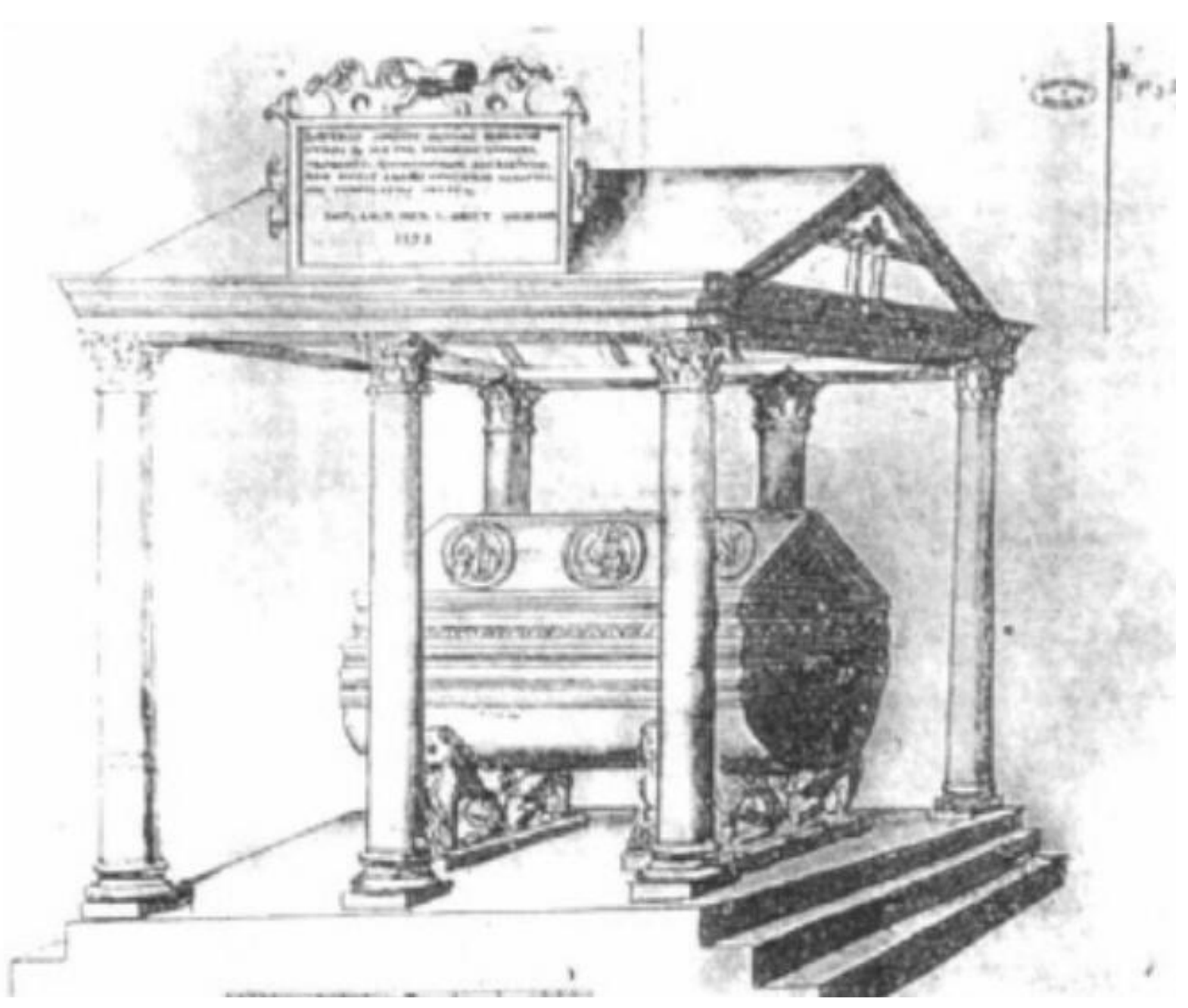

Fig. 9- Disegno allegato alla lettera di Diego Ortiz de Urizar con rappresentazione del sarcofago di Federico II (immagine da La Duca, 2002, 306).

7. IL PORFIDO ED IL NUOVO PANTHEON DELLA DINASTIA NORMANNA

Come Ruggero prima di lui, anche Guglielmo II provvede alla fondazione nel 1174 di una chiesa dinastica, con funzione di sepolcreto reale: Santa Maria la Nuova; anch'essa in una sede diversa da Palermo, ma questa volta limitrofa alla capitale, nella viciniore Monreale. Chiesa che in ragione del potere di Legato apostolico a latere innalza a cattedrale e sede arcivescovile fin dal 1183.

La creazione della diocesi di Monreale è in diretta polemica con lo "strapotere" dell'arcivescovo palermitano, che è riuscito ad imporre fra le proteste del capitolo cefaludense il mancato rispetto della volontà del re, quel Ruggero II che ha espresso il desiderio di essere sepolto a Cefalù. Stante il precedente sembra ragionevole che Guglielmo II abbia preferito una più vicina sede per la sepoltura propria e del padre, già tumulato nella cappella palatina.

Il disegno posto in essere da Guglielmo II mutua un altro locus della fenomenologia del potere bizantino legato agli spazi della sovranità nella morte. Si realizza un complesso progetto che prevede un tutt'uno fra la chiesa ed il palazzo reale. In tal modo Guglielmo II trasferisce in Sicilia un modello già presente a Costantinopoli. Il progetto rispecchia la consuetudine posta in essere anche per i SS. Apostoli, nei cui pressi sono annessi i cosiddetti oikoi basileioi. Ma vi è molto di più. Guglielmo II concepisce un complesso monumentale che prevede in continuità la chiesa dinastica, il convento dei benedettini e la sede regia. Questi vuole riproporre la situazione urbanistica che intercorre fra il 
Sacro Palazzo costantinopolitano e la cattedrale di S. Sophia. E se S. Sophia viene considerata la «Grande Chiesa», anche Monreale funge da grande chiesa di Stato, sostituendosi alla metropolia di Palermo. La fondazione di Monreale duplica il polo civile, costituito dal "Palazzo dei Normanni", e si oppone all'unico polo del potere religioso della capitale. Una duplicazione possibile soltanto in uno «Stato relativamente giovane» che si rivolge all'imitazione dell'Oriente cristiano (Krönig, 1981, 306). In tal modo si spiega la condotta del sovrano siciliano, che imita le strategie di auto-rappresentazione più autorevoli e le adegua al contingente con soluzioni che tengono in conto l'esperienza architettonica dell'Occidente.

Al fine di dare adeguata sepoltura a Guglielmo I, si pone nuovamente in essere il riutilizzo politicamente orientato di spolia di porfido; ciò avviene in una data non anteriore al 1183. Il modello è quello consueto: il sepolcro cefaludense, che reinterpreta il prototipo romano. L'arca è contraddistinta da un coperchio a spiovente con croci palmate iscritte nei profili cuspidati a sostituire la corona. La cassa al contrario si presenta con qualche velleità artistica, giacché decorata nei lati corti da due ghirlande con inscritta una foglia di edera. Il bordo è invece caratterizzato dal solito profilo ad ovuli. Il sarcofago a sua volta è posto su delle doppie mensole, ornate da modanature e dalle consuete scanalature. E se per la resa della cassa in un sol pezzo si può ipotizzare l'uso di una colonna di dimensione rilevante, si osserva come il coperchio sia realizzato in due tronconi,

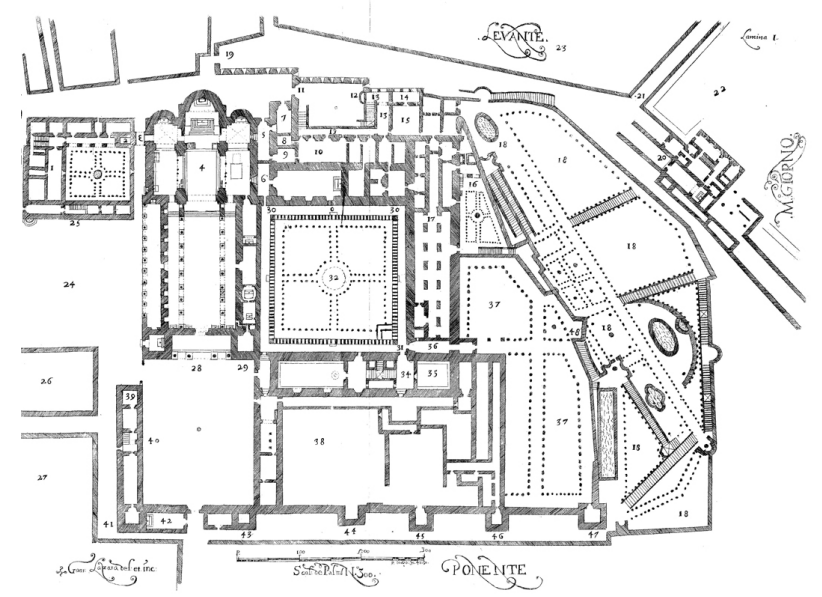

Fig. 10- Pianta del complesso di Monreale, Chiesa, Monastero e Palazzo reale (immagine da Delogu, 1983, 207, fig. 3). probabilmente con materiale di risulta. Lo stesso accade per le mensole che nella loro forma asimmetrica risultano quale soluzione che ripiega su sezioni dello stesso blocco. Un segnale che dimostra una prima difficoltà nel reperire il porfido per le commissioni reali; soluzione che apre a misure straordinarie che ottimizzano il materiale disponibile. Il sepolcro in origine è fornito di un baldacchino andato perso nell'incendio del 1811. Tuttavia resti di questo sono rinvenibili come materiale erratico nel chiostro, evidenze quali i capitelli a foglie piatte ma slanciate, assimilabili a quelle dei capitelli in porfido del santuario della stessa cattedrale; ciò rimanda alla mano comune di lapicidi specializzati. Si riscontra pure un nuovo tipo di resa dell'elemento vegetale, laddove la foglia si fa carnosa e compare persino il fiore; una progressione nella plastica dovuta all'esperienza accumulata dalle officine nell'elaborazione del materiale. A questo si sommano anche frammenti di basi dal gusto classico.

Gugliemo II come ben noto muore senza eredi. Tancredi altronde ha poco interesse a disporre una particolare sepoltura per quel predecessore che ha preferito combinare il matrimonio della zia, monaca da "smonacare", con l'imperatore.

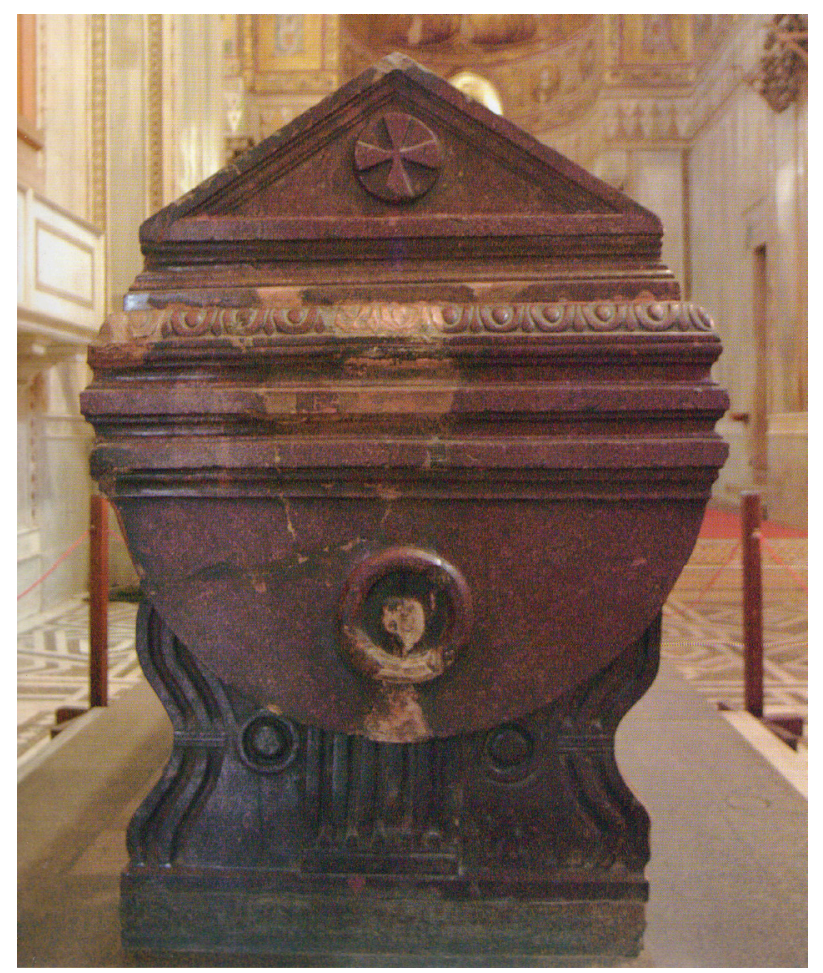

Fig. 11- Sepolcro di Guglielmo I, chiesa di Santa Maria la Nuova, Monreale (immagine da Gandolfo, 2006, 208, fig. 14). 
Ma non solo. Come Costantino prima di lui, anche Guglielmo immagina la chiesa di S. Maria la Nuova come un sacrario, laddove il suo corpo si presenta come reliquia insigne che viene a collocarsi ai piedi dell'altare. Una collocazione in verità ambigua, se si interpreta il gesto come segno di auto-magnificazione eccessivo, che si spiega nell'humus culturale altomedievale. Il corpo del sovrano che partecipa di una sorta di sacralità e santità d'ufficio diventa tout court cosa sacra e come tale merita una degna allocazione. La condotta diventa così plausibile solo dopo il gesto di Costantino, nonostante tale scelta non sia condivisa dal clero, che si trova a venerare durante la SS. Messa il corpo regio, volente o nolente. Eppure la scelta nasconde un locus di estrema umiltà. La tomba diviene oggetto di calpestio da parte del medesimo clero. Una sorta di trappola simbolica, che apre ad interpretazioni contrastanti. Come Costantino, nemmeno Guglielmo può riposare nella tomba che si è predisposto, dato che l'arcivescovo Ludovico I Torres, preso dallo spirito del Concilio di Trento, gli destina una più modesta sepoltura nei pressi del sepolcro del padre Guglielmo. Modesta per dimensioni e materiali: il comune marmo bianco, decorato con istoriazioni dal sapore rinascimentale. Sepolcro che per cronologia e stile non può essere considerato in questa sede.

\section{Conclusioni}

Si delinea una vera e propria antropologia del porfido, laddove l'alto valore simbolico coincide con gli altissimi costi di un materiale che non ha alcuna funzione primaria, oltre quella di indicatore dell'eccellenza del rango. Il riutilizzo va pensato in termini retorici, come argomentazione che suggerisce il carisma di un potere non derivato; un'idea che ritorna nelle laudes e nei panegirici.

Gli spolia di porfido sono un mezzo di comunicazione, di propaganda e persuasione; veicolano messaggi che devono essere compresi immediatamente. Questo perché si crede che il porfido può esprimere visivamente il mistero della regalità, tanto che il suo uso è sufficiente a presentificarlo. Dire che il porfido evoca la regalità è riduttivo e non illustra efficacemente le sue potenzialità. Non è solo un simbolo, ma un segno di status il cui senso è rivelato attraverso un processo mentale: è l'ipostasi della regalità, "congelata" nella pietra ed esternalizzata per essere compresa nell'immediato da chi la vede. Tale processo d'approccio al reale però non origina nelle strutture del pensiero del Tardoanti- co, ma bensì in quello del Medioevo. Si può allora parlare di una cognizione in fieri e di uno scarto semantico progressivo rispetto alla percezione del Tardoantico.

La spolia da cui è tratto non è un mero ricettacolo di un significato aggiunto, come lo è stato per la tarda antichità. La pietra è dotata di qualità che sottrae all'istituzione, per poi restituirle a chi la possiede. La sua mera proprietà legittima così le ambizioni ed il ruolo del possessore.

Un materiale la cui lavorazione vive nelle offcine regie e sopravvive attraverso l'uso ideologico, che sottolinea aspetti della liturgia del Palazzo. Il centro è la Cappella palatina, laddove il porfido viene impiegato per l'esedra presso cui si colloca il trono e per le rotae che, prima della ridefinizione del bildprogramm sotto Guglielmo I, hanno ornato le pareti di quella che è stata anche un'aula regia. È dunque la particolare esperienza della regalità siciliana che lo richiede. Lo reclama persino il trono collocato a Monreale e lo si esige necessariamente per i segnacoli di un potere che vuole sopravvivere all'evento morte.

La tecnica al contempo si affina, fino alla svolta verso soluzioni di ripiego, volte ad ottimizzare il poco materiale disponibile in epoca federiciana. Ciò conferma l'efficacia di un prodotto soggetto a copertura culturale, quale status symbol elitario solo appannaggio della corte, ma dotato di un alto quoziente di senso condiviso dalla società.

\section{BiBLIOGRAFIA}

Andaloro, M. (2002), "Per la conoscenza e la conservazione delle tombe reali della Cattedrale di Palermo: linee storiche e storico artistiche", Il sarcofago dell'imperatore. Studi, ricerche e indagini sulla tomba di Federico II nella Cattedrale di Palermo 1994-1999, Palermo, 135-148.

Barolo, M. T. (2002), "Federico II 'morto e vivente': significati della tomba nel Duomo di Palermo", Labyrinthos, XXI, 41/42, 3-23.

Carile, R.A. (2000), "Le insegne del potere a Bisanzio", La corona e i simboli del potere, Rimini, 65-124.

(2002), "La sacralità rituale dei $\beta \alpha \sigma \imath \lambda \varepsilon \tilde{\imath} \sigma$ bizantini”, Per me reges regnant. La regalità sacra nell'Europa medievale (Cardini, F. y Saltarelli, M. Eds.), Rimini-Siena, 53-95.

Cavallo, G. (1996), "La potenza della porpora”, Il Sole 24 Ore CCXCV. 
Catalano, G. (1965/1966), "Filippo II e le tombe dei Re di Sicilia”, Archivio Storico Siciliano, serie III, vol. 16, 179-190.

Daniele, F. (1784), I Regali sepolcri del Duomo di Palermo riconosciuti e illustrati, Napoli.

Delogu, P. (1973), "L'evoluzione politica dei Normanni d'Italia fra poteri locali e potestà universali”, Atti del Congresso internazionale di studi sulla Sicilia Normanna, Palermo, 4-8 dicembre 1972, Palermo, 51-104.

(1977), 'I Normanni in città. Schemi politici ed urbanistici, Società, potere e popolo nell'età di Ruggero II", Mito di una città meridionale: Salerno (Sec. VIII-IX), Napoli, 173-205.

(1983), "Idee sulla regalità: l'eredità normanna", Potere, società e popolo tra età normanna ed età sveva (1189-1210), Atti delle Quinte Giornate Normanno-Sveve, Bari-Conversano, 26-28 ottobre 1981, Bari, 185-214.

(1994), Introduzione allo studio della storia medievale, Bologna.

(1994), "La committenza degli Altavilla: produzione monumentale e propaganda politica", I Normanni popolo d'Europa. 1030-1200, Catalogo della Mostra, Roma, Palazzo Venezia, 28 gennaio-30 aprile 1994 (D’Onofrio, M. Ed.), Venezia, 188-192.

(1995), "Unio regni ad imperium": l'Italia meridionale e l'impero-germanico nel medioevo", Federico II e l'Italia. Percorsi, Luoghi, Segni e Strumenti, Catalogo della Mostra, Roma, Palazzo Venezia, 22 dicembre 1995 - 30 aprile 1996 (Fonseca, C.D. Ed.), Roma, 15-19.

De Champeaux, G. (1992), I simboli del medioevo, Milano.

Deér, J. (1959), The Dynastic Porphyry Tombs of the Norman Period in Sicily, Cambridge (Massachusetts).

Demus, O. (1947), Byzantine mosaic decoration, London.

(1988), The Mosaics of Norman Sicily, New York.

(2008), L'arte bizantina e l'Occidente, Torino.

Di Gregorio, R. (1821), Discorsi attorno alla Sicilia, Palermo.

D’Onofrio, M. Ed. (1994), I Normanni popolo d'Europa. 1030-1200, Catalogo della Mostra, Roma, Palazzo Venezia, 28 gennaio-30 aprile 1994, Venezia, XX-590.

(1994), "Il panorama dell'architettura religiosa", I Normanni popolo d'Europa. 1030-1200,
Catalogo della Mostra, Roma, Palazzo Venezia, 28 gennaio-30 aprile 1994 (D’Onofrio, M. Ed.), Venezia, 199-207.

Downey, G. (1959), "Le tombe degli imperatori bizantini presso la Chiesa dei Santi Apostoli a Costantinopoli”, Journal of Hellenic Studies, LXXIX, 27-51.

Duby, G. (1985), L'arte e la società medievale, Torino.

Dupont, F. (1986), Le corps des dieux: le temps de la reflexion, Paris.

(1986), "L'autre corp de l'empereur-dieu", Le temps de la réflexion, VII, 231-252.

Faedo, L. (1982), "La sepoltura di Ruggero Gran Conte di Calabria", АПАРХАI: Nuove ricerche e studi sulla Magna Grecia e la Sicilia antica, Studi in onore di P.E. Arias, Pisa, 691-706.

Gandolfo, F. (2006), "Il porfido," Nobiles Officinae: perle, filigrane e trame di seta dal Palazzo Reale di Palermo (Vol. 1-2) (Adaloro, M. Ed.), Catania, 425-429.

Giunta, F. (1974), Bizantini e bizantinismo della Sicilia normanna, Palermo.

(1981), "Il Regno tra realtà europea e vocazione mediterranea”, Potere, società e popolo nell'età dei due Guglielmi, Atti delle Quarte Giornate Normanno-Sveve, Bari -Gioia del Colle, 8-10 ottobre 1979, Bari, 9-29.

Grierson, P.H. (1962), “The Tombs and Obits of the Byzantine Emperors (337-1042)”, Dumbarton Oaks Papers, XVI, 3-60.

Grierson, P.H. y Mango, C. (1962), “The Tombs and Obits of the Byzantine Emperors (337-1042). With an Additional Note", Dumbarthon Oaks Paper, XVI, 1-63.

Kantorowicz, E. (1989), I due corpi del Re. L'idea di regalità nella teologia politica medievale, Torino.

Krönig, W. (1973), "Vecchie e nuove prospettive sull'arte della Sicilia normanna", Atti del Congresso Internazionale di Studi sulla Sicilia Normanna, Palermo, 4-8 dicembre 1972, Istituto di Storia Medievale Università di Palermo, Caltanissetta-Roma, 132-145.

(1981), "Sul significato storico dell'arte sotto i due Guglielmi", Potere, società e popolo nell'età dei due Guglielmi, Atti delle Quarte Giornate Normanno-Sveve, Bari-Gioia del Colle, 8-10 ottobre 1979, Bari, 291-310.

Herklotz, J. (1985), “'Sepulcra' e 'monumenta' del 
medioevo". Studi sull'arte sepolcrale in Italia, Collana di studi di storia dell'arte, V, Roma.

(1994), "Lo spazio della morte e lo spazio della sovranità", I Normanni popolo d'Europa. 1030-1200, Catalogo della Mostra, Roma, Palazzo Venezia, 28 gennaio-30 aprile 1994 (D’Onofrio, M. Ed.), Venezia, 320-326.

La Duca, R. (2002), "Una cattedrale per Federico. Vicende Storiche del sarcofago di Federico II", Il sarcofago dell'imperatore. Studi, ricerche e indagini sulla tomba di Federico II nella Cattedrale di Palermo 1994-1999, Palermo, 303-315.

Marcone, A. (2002), Pagano e cristiano. Vita e mito di Costantino, Roma-Bari.

Molina Lopez, L. (2011), "Viaje a Italia a traves de las Cantigas Historiadas de Alfonso X el Sabio", Saberes Artisticos bajo signo y designios del Urbinate, Anales de Historia del Arte, Madrid, 319-330.

(2014), "La valorizzazione e diffusione del modello delle Tombe Regali di Palermo nella Penisola Iberica", L'Impero e le Hispaniae da Traiano a Carlo V. Classicismo e potere nell'arte spagnola (De Maria Manuel, S. y López De Corselas, P. Eds.), Bologna, 56-64.

Mongitore, A. (1873), La Cattedrale di Palermo, Palermo.

Morrone, M. (1998), "L'antico nella Calabria medievale fra architettura di prestigio e necessità", Mélanges de l'Ecole de Rome, Moyen Age CX, 1, 341-357.

Paravicini Bagliani, A. (1998), Le Chiavi e la Tiara. Immagini e simboli del papato medievale, Roma.

Pensabene, P. (1990), "Contributo per una ricerca sul reimpiego e il recupero dell'antico nel medioevo. Il reimpiego nell'architettura normanna”, RIA, XIII, 5-10.

Perea Yébenes, S. (2005), "Imago imperatoris, ad sidera! El funeral de los emperadores romanos, la apoteosis y el 'cuerpo doble", Oppidum I, 103-120.

Perricone, E. (1916), La tomba porfirea di Ruggiero II, Palermo.

Pertusi, A. (1964), "Bisanzio e l'irradiazione della sua civiltà in Occidente nell'Alto Medioevo", CISAM, XI Settimana di Studio, Centri e vie di irradiazione della civiltà nell'Alto Medioevo, Spoleto, 75-134.
(1976), "Insegne del potere sovrano e delegato a Bisanzio e nei paesi di influenza bizantina", CISAM, XIII Settimana di Studio, Simboli e simbologia nell'Alto Medioevo, Spoleto, 481568.

(1977), “Teoria del pensiero politico”, La civiltà bizantina dal IV al IX secolo, Bari, 31-85.

(1983), Storia delle idee politiche economiche e sociali (Firpo, L. Ed.), Torino.

(1991), Il pensiero politico bizantino (Carile, R. A. Ed.), Bologna.

Ramata, G. A. (1955), "I sarcofagi donati da Ruggero II alla chiesa di Cefalù e trasportati a Palermo per ordine di Federico II", Archivio Storico Siciliano, serie III, vol. 7, Palermo, 257-276.

Renda, F. (2012), Federico II e la Sicilia, Catanzaro.

Valenziano, C. y Valenziano, M. (1979), La basilica cattedrale di Cefalu nel periodo normanno, Palermo.

Vergara Caffarelli, F. (2002), "Fonti documentarie per la storia delle tombe reali", Il sarcofago dell'imperatore. Studi, ricerche e indagini sulla tomba di Federico II nella Cattedrale di Palermo 1994-1999, Palermo, 316-340.

Tronzo, W. (2011), "Il porfido nel Medioevo: racconti subliminali di un materiale", Seminario, Università Roma Tre, Roma. 
